\title{
The influence of Antarctica on the momentum budget of the southern extratropics
}

Article

Published Version

Juckes, M. N., James, I. N. and Blackburn, M. (1994) The influence of Antarctica on the momentum budget of the southern extratropics. Quarterly Journal of the Royal Meteorological Society, 120 (518). pp. 1017-1044. ISSN 1477870X doi: https://doi.org/10.1002/qj.49712051811 Available at https://centaur.reading.ac.uk/34989/

It is advisable to refer to the publisher's version if you intend to cite from the work. See Guidance on citing.

Published version at: http://dx.doi.org/10.1002/qj.49712051811

To link to this article DOI: http://dx.doi.org/10.1002/qj.49712051811

Publisher: Royal Meteorological Society

All outputs in CentAUR are protected by Intellectual Property Rights law, including copyright law. Copyright and IPR is retained by the creators or other copyright holders. Terms and conditions for use of this material are defined in the End User Agreement.

www.reading.ac.uk/centaur 
Central Archive at the University of Reading

Reading's research outputs online 


\title{
The influence of Antarctica on the momentum budget of the southern extratropics
}

\author{
By M. N. JUCKES*, I. N. JAMES and M. BLACKBURN \\ University of Reading, $U K$
}

(Received 22 December 1992; revised 26 August 1993)

\begin{abstract}
SUMMARY
The antarctic plateau acts as a strong heat sink for the global climate, cooling the atmosphere and radiating energy to space. A cold dense atmospheric boundary layer is formed. Strong surface winds are formed as the boundary layer drains off the plateau. These drainage winds and the eddy fluxes necessary to maintain them are analysed in a general circulation model (GCM). The drainage flow is well represented in the GCM. The associated mean meridional circulation is analysed in isentropic coordinates. The momentum budget over Antarctica reveals a balance between the Eliassen-Palm flux convergence and the Coriolis torque exerted by the mean meridional mass flux. Both vertical and horizontal components of the Eliassen-Palm flux contribute, the vertical component being the greater.
\end{abstract}

\section{INTRODUCTION}

The drainage flow of cold air off the antarctic plateau is a prominent feature of the climatology of the region (Schwerdtfeger 1984). Intense cooling at the ice surface (especially during winter) creates a strong inversion in the lowest few hundred metres of the atmosphere and the cold dense air drains off the continent. The shallow inversion layer contains winds of remarkable strength and steadiness. These drainage winds become stronger around the periphery of the ice sheet and are especially concentrated into particular localities, such as the vicinity of Cape Denison (see, for example, Parish 1982; Parish and Bromwich 1986, 1987; Bromwich et al. 1990).

The flow over the ice sheet interior has been analysed by Ball (1960). He found that the essential features of the drainage flow could be represented by a one-dimensional model in which a balance exists between the downslope component of the buoyancy force, which drives the flow; the viscous diffusion of momentum into the surface, which retards it; and the Coriolis force, which deflects it. Viscous diffusion in this model represents the effects of small-scale turbulence. The theory predicts that the velocity vector will not be parallel to the local fall line, but turned anticlockwise from the line of steepest descent. Such a turning of the wind is amply demonstrated by direct observations (references cited in the previous paragraph) and by indirect assessments of the mean wind direction provided by sastrugi orientations (Bromwich et al. 1990). However, Egger (1985) and James (1989) showed that in a zonally symmetric atmosphere the downslope component of the surface flow would fall to small values, much smaller than those typically observed, within a few days. Starting with an atmosphere at rest, they found that imposed strong surface cooling on the slopes of Antarctica initially generated a strong buoyancy-driven drainage flow. The upper tropospheric convergence over the pole, required by continuity to balance the divergence in the inversion layer, generated a cyclonic vortex centred over the pole. The resulting pressure gradient opposed the downslope component of the buoyancy forces, greatly reducing the drainage flow after a few days integration. In contrast to these idealized experiments, the observed drainage flow persists throughout the year. The observed persistence suggests that eddy momentum fluxes in the atmosphere provide a forcing which limits the intensity of the polar cyclonic vortex.

* Corresponding author. Present address: Meteorologisches Institut der Universität München, Theresienstrasse 37, 80333 München 2, Germany. 
James (1989) suggested a number of possibilities for providing the necessary momentum flux. These included the effective drag resulting from breaking internal gravity waves around the rugged periphery of Antarctica, and the effect of decaying mid-latitude weather systems, which tend to spiral into the circumpolar trough. Whichever of those mechanisms is most important, the situation is analogous to the maintenance of the mean meridional circulation in the stratosphere. There breaking Rossby waves generate a timemean diabatic circulation and keep the atmosphere out of thermal equilibrium (e.g. Andrews and McIntyre 1976; Dunkerton et al. 1981).

The paucity of observations over Antarctica, especially over the interior of the ice sheet, make detailed budget studies difficult. In this paper we examine the winter circulation in the vicinity of Antarctica as simulated by the UK Universities Global Atmospheric Modelling Programme (UGAMP) global circulation model (UGCM). The UGCM provides a qualitatively realistic simulation of the observed surface circulation over the antarctic ice sheet, including a strong surface inversion and a persistent strong pattern of surface drainage winds. The momentum balance of the upper polar vortex will be examined to determine what mechanisms provide the vorticity flux (or zonal momentum forcing) which allows the surface drainage regime in the model to persist. We shall show that vertical stresses exerted by synoptic-scale eddies provide the dominant term to balance the momentum forcing of the drainage flow.

The results in this paper have been analysed largely on isentropic surfaces, after the fashion of Townsend and Johnson (1985). Isentropic coordinates are ideal for analysing the meridional mass flux, since the mean meridional circulation in these coordinates agrees well with parcel trajectories. In contrast, the Eulerian zonal-mean meridional velocity in pressure coordinates is not necessarily even in the same sense as the mean parcel motion. The difference is related to the correlation between vertical displacements and eddy meridional velocities. In isentropic coordinates the vertical displacements are much smaller, because the motions are generally nearly adiabatic on synoptic time-scales, and hence the discrepancy between Eulerian and Lagrangian mean velocities is reduced. Synoptic-scale motions are more closely adiabatic at high latitudes than for the hemisphere as a whole, because the heating due to latent-heat release in synoptic weather systems is both limited and localized. In the mid-latitude storm belts such heating is an important component of the synoptic- and large-scale dynamical balances (Hoskins and Valdes 1990). Time-mean diagnostics in the isentropic system give a clean split in the momentum budget between a zonal-mean diabatic component and the eddy fluxes. A drawback of the isentropic system is that the earth's surface is not even approximately a coordinate surface. This problem also occurs in pressure coordinates, particularly in the vicinity of high orography, such as the antarctic plateau. The problem can be dealt with elegantly in isentropic coordinates using a method developed by Lorenz (1955) and Andrews (1983).

Iwasaki (1992) used similar diagnostics to analyse data from the National Center for Atmospheric Research (NCAR) Community Climate Model 1. The present work concentrates more on the antarctic region, and analyses the momentum budget in greater detail.

\section{THE MODEL}

The UGCM is derived from the European Centre for Medium-range Weather Forecasts (ECMWF) operational forecast model in the form used in July 1986 (cycle 27). The medium- and extended-range forecast behaviour of the ECMWF model has been studied by Tiedtke et al. (1988) and Palmer et al. (1990). The latter paper compared 
several versions of the model, amongst which the one used operationally in the northern hemisphere winter of $1986 / 7$ most closely resembles the UGCM as used here. The only significant difference between that version of the ECMWF model and the UGCM is in the parametrization of lateral dissipation (described later).

The principal integration studied here employs a spectral truncation at total wave number 42 (T42), equivalent to a horizontal wavelength of roughly $1000 \mathrm{~km}$. The hybrid pressure-sigma vertical coordinate is that introduced by Simmons and Burridge (1981), with 19 levels up to $1 \mathrm{kPa}$ (near $30 \mathrm{~km}$ ). The vertical coordinate, $\eta$, is defined such that

$$
p(\eta)=A(\eta)+B(\eta) p_{\mathrm{s}}
$$

where $p$ is the pressure and $p_{\mathrm{s}}$ the surface pressure. $A$ and $B$ are prescribed functions such that at the top of the model the model surfaces are constant pressure $(B=0)$, whilst the lower levels follow the orography $(A=0)$. Over Antarctica the heights of the lowest three leveis, which contain most of the drainage flow, are approximately 30, 140 and $360 \mathrm{~m}$.

The model is run through a seasonal cycle with prescribed sea surface temperatures (SST), the monthly climatology of Alexander and Mobley (1976) being interpolated smoothly in time, beginning from January. The period analysed is the first southern hemisphere winter, 6 months into the run. The sea-ice distribution is prescribed from the SST. Surface albedo is a prescribed annual mean.

The time integration scheme is semi-implicit with a 30 -minute time step. Both the resolved linear gravity waves and the advection due to the zonal mean flow are treated implicitly.

The radiation scheme was developed at the ECMWF and is described by Morcrette (1990), where the version used here is referred to as 'OPE'. It is known to underestimate both clear-sky cooling and the infrared effects of cloud, resulting in insufficient radiative destabilization of the atmosphere. Clouds are determined from the humidity and stability properties in each column, as described by Slingo (1987). Kuo's parametrization of penetrative convection is coupled with a diffusive formulation of shallow convection, both described by Tiedtke $e t$ al. (1988). A parametrization of large-scale rain removes grid-scale supersaturation. Vertical diffusion is included throughout the depth of the atmosphere with fluxes calculated from Monin-Obukhov similarity theory. Surface fluxes use bulk aerodynamic formulae. A parametrization of gravity-wave drag is included, following Palmer et al. (1986).

The time-scale of lateral dissipation is based on a study of linear and nonlinear baroclinic growth in a spectral model reported by MacVean (1983). $A \nabla^{6}$ hyperdiffusion is used with a time-scale of 4 hours at the truncation limit. The time-scale is reduced at the uppermost model levels, halving at each level above $10 \mathrm{kPa}$ to reach 15 minutes at $1 \mathrm{kPa}$. This is necessary to prevent instability which would otherwise occur when the Courant-Friedrichs-Lewy condition for numerical stability is violated in the region of the stratospheric polar-night jet.

The model was initialized with the ECMWF analysis for 15 January 1987 . The results discussed later come from days 138-234 of the experiment. The issues of interannual variability and the spin-up of the model are not addressed here. The emphasis is on understanding the mechanisms which maintain the model circulation on the seasonal time-scale. For these purposes it is sufficient to have a model that can reproduce the principal features of the flow in question over the desired length of time. The adjustment time-scale for the drainage flow in the simple models that motivated this study was of the order of a few days, much shorter than the spin-up time allowed in the present experiment. 
No attempt has been made to tune the model parametrization schemes to the extreme conditions of Antarctica. The analysis presented concentrates on the large-scale dynamical aspects of the drainage flow. These processes are not directly dependent on the details of the parametrization schemes.

\section{ISENTROPIC DIAGNOSTICS}

In isentropic coordinates the zonal-momentum equation can be written (e.g. Andrews et al. 1987):

$$
\frac{\partial u}{\partial t}+\mathbf{v} \cdot \nabla u-\left(f+\frac{u \tan \phi}{a}\right) v+\frac{1}{a \cos \phi} \frac{\partial M}{\partial \lambda}=X
$$

where $M=c_{p} T+\Phi$ is the Montgomery potential and $X$ represents all parametrized terms, including vertical advection. The remaining terms are standard notation as follows: $v=(u, v)$ is the horizontal velocity in the latitude $(\phi)$ longitude $(\lambda)$ coordinate system, $f$ is the Coriolis parameter, $a$ the earth's radius, $T$ is temperature, $c_{p}$ the specific heat of air at constant pressure and $\Phi$ is the geopotential height.

The hydrostatic equation becomes

$$
\frac{\partial M}{\partial \theta}=\frac{c_{p} T}{\theta}
$$

where $\theta$ is the potential temperature.

The density in isentropic coordinates is given by

$$
\sigma=-g^{-1} \frac{\partial p}{\partial \theta}
$$

where $g$ is the acceleration due to gravity. With this definition the mass of air in a volume $V$ is given by $\int_{V} \sigma \mathrm{d} x \mathrm{~d} y \mathrm{~d} \theta$.

Mass-weighted means are used, as described by Tung (1986). An overbar will be used for the zonal average,

$$
\bar{\sigma}=\frac{1}{2 \pi} \int_{0}^{2 \pi} \sigma \mathrm{d} \lambda
$$

The mass-weighted mean is then defined as

$$
\hat{u}=\frac{\overline{\sigma u}}{\bar{\sigma}}
$$

and the departure from the mean is $u^{*}=u-\hat{u}$.

Where potential-temperature surfaces intersect the ground, the convention devised by Lorenz (1955) is followed. This allows zonal means to be calculated without introducing any spurious unphysical contributions. The isentropic surfaces are assumed to continue below the ground, with $p$ equal to $p_{\mathrm{s}}$. The pressure is then independent of $\theta$ below ground, so that $\sigma$ is identically zero (Eq. (4)). It follows that the mass-weighted means contain no contribution from areas where the isentropes are beneath the earth's surface.

The eddy terms in the momentum equation can be expressed as the divergence of the Eliassen-Palm flux (EP flux). This diagnostic was first developed in pressure coordinates by Andrews and McIntyre (1976), building on earlier work by Eliassen and Palm. The form used here in isentropic coordinates closely follows that derived by Andrews 
(1983). Andrews used the zonal mean $\overline{(~)}$ and departures from that mean, whereas, as noted earlier, the mass-weighted mean is used here.

Combining the zonal-momentum equation with the mass-conservation equation, and taking the zonal mean gives

$$
\frac{\partial \hat{u}}{\partial t}-\hat{v}\left(f-\frac{1}{a \cos \phi} \frac{\partial}{\partial \phi}(\hat{u} \cos \phi)\right)=\frac{1}{\bar{\sigma} \cos \phi} D+\hat{X}
$$

where $D$ is the EP-flux divergence, given by:

$$
D=\frac{1}{\bar{\sigma} \cos \phi}\left(\frac{1}{a \cos \phi} \frac{\partial\left(F_{\phi} \cos \phi\right)}{\partial \phi}+\frac{\partial F_{\theta}}{\partial \theta}\right) .
$$

The horizontal and vertical components of the flux are given by

$$
\mathbf{F}=\left(F_{\phi}, F_{\theta}\right)=\left\{-\bar{\sigma} \hat{u * v} * \cos \phi, \frac{1}{g a} \overline{\left(p \frac{\partial M}{\partial \lambda}\right)}\right\}
$$

These quantities are closely related to the more widely used pressure-coordinate version. Consider a zonally symmetric reference state, $\theta_{\mathrm{r}}, p_{\mathrm{r}}$. For small departures from this reference state the pressure perturbation, $p^{\prime}$, on the isentropic surface is related to the potential-temperature perturbation, $\theta^{\prime}$, on the isobaric surface by

$$
p^{\prime} \approx-\frac{\partial p_{\mathrm{r}}}{\partial \theta_{\mathrm{r}}} \theta^{\prime}
$$

Combining (10) with the definition of the geostrophic wind,

$$
f v_{\mathrm{g}}=\frac{1}{a \cos \theta} \frac{\partial M}{\partial \lambda}
$$

gives

$$
F_{\theta} \approx \frac{-\overline{f v^{\prime} \theta^{\prime}} \cos \phi}{\partial \theta_{\mathrm{r}} / \partial p_{\mathrm{r}}}
$$

which is the vertical component of the geostrophic EP flux in pressure coordinates, with a minus sign because this flux is positive in the direction of increasing $\theta$ instead of pressure.

The isentropic coordinate formulation is particularly attractive over Antarctica because of the elegant way in which the large-amplitude orography can be handled. The pressure-coordinate fluxes become ill-defined when the pressure surfaces intersect the orography but, using the formalism developed by Andrews (1983), the isentropic fluxes are well defined. Andrews also shows that the vertical component of the EP flux has a simple physical interpretation. Namely, it is the form drag that an isentrope exerts on the atmosphere above. At ground level this becomes the pressure torque exerted by the earth's orography on the atmosphere.

The principal advantage of the isentropic analysis over the transformed-Eulerianmean (TEM) equations (Andrews and McIntyre 1976) is the clean treatment of the lower boundary. Another advantage is the more direct portrayal of the diabatic heating, which is an essential part of the mean meridional motion.

In the TEM equations an eddy term, representing the Stokes drift, is added to the zonal-mean meridional velocity to give a velocity which represents material motion more closely. A similar dual perspective is useful in the isentropic framework. The following is based on a division of the mass flux into geostrophic and ageostrophic components, a 
diagnostic approach introduced by Johnson (1989). The geostrophic component is given by:

$$
f \overline{v_{\mathrm{g}} \sigma}=\frac{1}{a \cos \phi} \overline{\left(\frac{\partial p}{\partial \lambda}\right)_{\Phi} \sigma}=-\frac{1}{\cos \phi} \frac{\partial F_{\theta}}{\partial \theta}
$$

where $v_{\mathrm{g}}$ is the geostrophic velocity. This relation shows that the effective force due to any vertical flux of momentum will be balanced by that due to the geostrophic mass flux. Using (12) the pressure force can be eliminated from the momentum equation, leaving

$$
\frac{\partial \hat{u}}{\partial t}+\frac{1}{a \bar{\sigma} \cos ^{2} \phi} \frac{\partial}{\partial \phi}\left(\hat{\sigma} \hat{u} \widehat{b} * \cos ^{2} \phi\right)-f \hat{v}_{\mathrm{ag}}+\hat{v}\left(\frac{1}{a \cos \phi} \frac{\partial}{\partial \phi}(\hat{u} \cos \phi)\right)=\hat{X}
$$

where

$$
f \hat{v}_{\mathrm{ag}}=f \hat{v}-f v_{\mathrm{g}}
$$

Equations (12-14) are valid globally, with no restrictions on the value of $f$. When $f$ is small, however, the physical interpretation of $\hat{v}_{\mathrm{ag}}$ is unclear. In particular, $f \hat{v}_{\mathrm{ag}}$ does not necessarily vanish at the equator. This is not a problem in the extratropics. Equation (13) now has the same structure as the pressure coordinate zonal-mean momentum equation, with $f v$ in the latter replaced by $f \hat{v}_{\text {ag }}$ in (13).

The mass equation can be written

$$
\frac{\partial \bar{\sigma}}{\partial t}+\frac{1}{a \cos \phi} \frac{\partial}{\partial \phi}\left(\bar{\sigma} \hat{v}_{\mathrm{ag}}+\frac{1}{f \bar{\sigma} \cos \phi} \frac{\partial F_{\theta}}{\partial \theta}\right)+\frac{\partial \overline{\sigma^{2}}}{\partial \theta}=0
$$

where 2 is the heating rate. This equation now resembles the pressure-mean thermodynamic equation, in which mean advection and eddy heat fluxes (represented by $F_{\theta}$ through Eq. (11)) balance the diabatic heating.

The similarity between the equations governing the ageostrophic circulation in isentropic coordinates and those for the Eulerian mean circulation in pressure coordinates suggest that the two circulations should have similar structure. Similarly, the isentropic mass-weighted meridional circulation can be expected to resemble the TEM circulation. These expectations have been confirmed from observational data by Johnson (1989). Each method of dividing the flow into mean and eddy components has its advantages, which depend to some extent on the flow in question. The choice of representation depends, in part, on the relative amplitudes of $\hat{v}$ and $\hat{v}_{\text {ag }}$. In stratospheric applications the TEM circulation is smaller than the Eulerian mean (Edmon et al. 1980); using the former gives a clearer picture of the dynamics. In the extratropical troposphere, in contrast, $\hat{v}$ is larger than $\hat{v}_{\text {ag }}$ (Johnson 1989). This suggests that the transformed equations ((13)-(15)) might give a clearer picture of the momentum budget, with the large cancellation between vertical momentum fluxes and the Coriolis torque due to the geostrophic mass flux eliminated from the equations.

The difference in behaviour between the stratospheric and tropospheric circulations is probably due both to the difference between the static stabilities of the two regions, and to the different forcing distributions. The properties of the response of such a system to forcing, given here by the EP-flux divergence, have been analysed by Eliassen (1951) and Plumb (1979). The response in the residual mean meridional circulation is proportional to the vertical gradient of the forcing and inversely proportional to the static stability. Both factors imply weaker meridional motions in the stratosphere, where, relative to the troposphere, the static stability is larger and the vertical scale of the forcing typically greater. Where the static stability is large the response to a given forcing 
will be mainly in the zonal-mean zonal velocity, with small associated changes in the temperature. In this case the diabatic circulation $\hat{v}$ will be small compared to $\bar{v}$. When the static stability is small the response will be larger in the temperature field, so that $\hat{v}$ may be larger than $\bar{v}$.

There is one drawback in the use of $\hat{v}_{\mathrm{g}}$ and $\hat{v}_{\mathrm{ag}}$ : the two circulations do not independently conserve mass. This feature is independent of the coordinate system used. In pressure coordinates the geostrophic velocity vanishes when averaged around any pressure surface that does not intersect the ground, but gives non-zero values when averaged along partial surfaces. The net geostrophic mass flux across any latitude is equal to the orographic form drag:

$$
\int_{0}^{p_{\mathrm{s}}} \mathrm{d} p \int_{0}^{2 \pi} \mathrm{d} \lambda\left(f v_{\mathrm{g}}\right)=-\int_{0}^{2 \pi} \mathrm{d} \lambda\left(p \frac{\partial \Phi_{\mathrm{s}}}{\partial \lambda}\right)
$$

where $\Phi_{\mathrm{s}}$ is the geopotential height of the orography. In the time mean this mass flux is balanced by an equal and opposite ageostrophic mass flux.

A zonal-mean formulation similar to that used here has been used by Iwasaki (1989); namely hybrid pressure-isentrope coordinates $(p \dagger)$ and a transformed-Eulerian-mean formulation for those coordinates ( $p \nmid-T E M)$. Iwasaki uses isentropic averages, with mass weighting as used here, but then uses the zonal mean pressure $\bar{p}(\theta)$ as the vertical coordinate in place of $\theta$. The main difference between the $p \nmid-$ TEM equations and those used here is that the former have a non-zero adiabatic vertical velocity, which generates an additional term in the vertical EP flux. When $\theta$ is used as the vertical coordinate the vertical motion is purely diabatic. The $p \nmid-T E M$ approach gives equations that are closer to the TEM equations, thus facilitating comparison with pressure-coordinate diagnostics.

For horizontal distributions on the hemispheric scale, isentropic surfaces are not ideal because surfaces which are in the upper troposphere at the poles intersect the ground near the tropics. Hence they pass through a variety of different dynamical regimes. For this reason a combination of isentropic and isobaric maps will be used below.

\section{NUMERICAL INTERPOLATION}

Care must be taken when interpolating results from the model grid onto another coordinate system. The model fields tend to have a lot of structure down to the gridscale, so the results can be sensitive to the form of interpolation used. The next few paragraphs describe the method used. The levels used are chosen to be roughly equally spaced in pressure. This is done by having equal steps in $\mathscr{P}$, where $\mathscr{P}$ is defined by

$$
\mathscr{P}=\left(\frac{\theta}{T_{00}}\right)^{-3.5}
$$

where $T_{00}$ is a constant reference temperature.

In an isothermal atmosphere, with $T=T_{00}$, the vertical coordinate approximates the pressure, a small difference arising from the use of 3.5 in place of $c_{p} /\left(c_{p}-c_{v}\right)$, where $c_{v}$ is the specific heat of air at constant volume.

Full levels are defined by

$$
\mathscr{P}_{k}=\mathscr{P}_{\min }+\frac{k-1}{N-1}\left(\mathscr{P}_{\max }-\mathscr{P}_{\min }\right), \quad k=1, \ldots, N
$$

where $\mathscr{P}_{\max }$ and $\mathscr{P}_{\min }$ correspond to $\theta=225$ and $450 \mathrm{~K}$ respectively. 
Pressure is interpolated onto half levels, defined by

$$
\left.\begin{array}{c}
\theta_{k+1}=\frac{\theta_{k}+\theta_{k+1}}{2} \quad 1 \leqslant k \leqslant N-1 \\
\theta_{N+1}=2 \theta_{N}-\theta_{N-1} \\
\theta_{1}=\min \left(\theta_{\mathrm{s}}\right) .
\end{array}\right\}
$$

Using a staggered grid in this way improves the accuracy with which the density, $\sigma$, is calculated.

This paper examines mass and momentum fluxes. It is, therefore, essential to ensure that the interpolation onto isentropic surfaces does not introduce spurious fluxes of these quantities. The mean meridional motion and the divergence of $F_{\theta}$ need particular care because they are both small residuals from sums of large terms. Spurious fluxes are avoided by interpolating the vertical integrals of these two quantities.

The algorithm is described here for a general quantity $Q$, which could be the meridional velocity $v$ or the pressure-gradient term in the momentum equation, $\partial M / \partial \lambda$.

The vertical integral,

$$
S_{Q}\left(p_{k}\right)=\int_{p_{k}}^{p_{\mathrm{s}}} Q(p) \mathrm{d} p
$$

is calculated at each model level and then interpolated onto $\theta$ coordinate half levels, giving $\left\{S_{Q}\left(\theta_{k+\downarrow}\right): k=0, \ldots, N\right\}$. The value of $Q$ on full levels is then obtained by

$$
Q\left(\theta_{k}\right)=\frac{S_{Q}\left(\theta_{k+1}\right)-S_{Q}\left(\theta_{k-1}\right)}{p\left(\theta_{k+1}\right)-p\left(\theta_{k-\frac{1}{2}}\right)}
$$

It follows that

$$
\widehat{Q}\left(\theta_{k}\right)=\frac{\overline{S_{Q}\left(\theta_{k+1}\right)}-\overline{S_{Q}\left(\theta_{k-1}\right)}}{\Delta \bar{p}}
$$

and

$$
\int \widehat{Q\left(\theta_{k}\right)} \mathrm{d} \bar{p}=\overline{S_{Q}\left(\theta_{\max }\right)}-\overline{S_{Q}\left(\theta_{\min }\right)}
$$

Hence, if the integral $S_{Q}$ (Eq. (21)) is consistent with the model finite differencing the same is true of the mass-weighted mean in Eq. (24). This method ensures that no spurious sources of $Q$ are generated in the interpolation.

Taking $\rho$ as the density the gradient of the Montgomery potential is related to quantities calculated in the model by

$$
\left(\frac{\partial M}{\partial \lambda}\right)_{\theta}=\rho^{-1}\left(\frac{\partial p}{\partial \lambda}\right)_{\Phi}=\rho^{-1}\left(\frac{\partial p}{\partial \lambda}\right)_{\eta}+\left(\frac{\partial \Phi}{\partial \lambda}\right)_{\eta}
$$

The right-hand side of $(25)$ is evaluated using the model finite-difference scheme. This procedure prevents spurious momentum sources being introduced. One drawback is that, after interpolation, $\overline{\partial M / \partial \lambda}$ is not in general zero, whereas it clearly should be. For this study the global momentum conservation is felt to be more important than the above problem.

The model fields are saved every 18 hours. This period was chosen to reduce aliasing of the diurnal cycle onto time-mean diagnostics (Thurburn 1991). 


\section{THE MEAN CIRCULATION}

The model has a persistent drainage flow of considerable strength. Figure 1 shows the model drainage flow, averaged over June, July and August. The Greenwich meridian is to the right of this and all the other stereographic plots. The arrows show the wind speed on the lowest model level $(A=0 \mathrm{kPa}, B=0.9961$ in Eq. (1), about $30 \mathrm{~m}$ above the surface). The maximum winds, $16 \mathrm{~m} \mathrm{~s}^{-1}$, are somewhat less than the observed maximum, presumably because the lowest model layer is too thick to resolve the flow fully. The winds around the edge of the continent are at roughly $45^{\circ}$ to the height contours. Other GCMs produce a similar pattern (James 1993). This pattern is consistent with the analytic work of Ball (1960), in which frictional forces balance the Coriolis and buoyancy forces. The results discussed in the next section suggest that, despite the agreement shown here, there are significant differences between the balance of forces in the UGCM and that in the Ball model. In the Ball model frictional forces balance the Coriolis term, but in the UGCM large-scale pressure gradients also play a major role. The constancy of the wind (defined as the speed of the mean wind divided by the mean of the wind speed) is of the order 0.8 to 0.9 where the orography is strongly sloping. Similar values are found in observations (Schwerdtfeger 1984).

The drainage flow is associated with a strong temperature inversion, whose strength and depth are illustrated in Figs. 2(a) and 2(b) respectively. The top of the inversion is taken to be the first maximum in the temperature profile. The strength is the difference between the temperature at the top of the inversion and at ground level. The averages

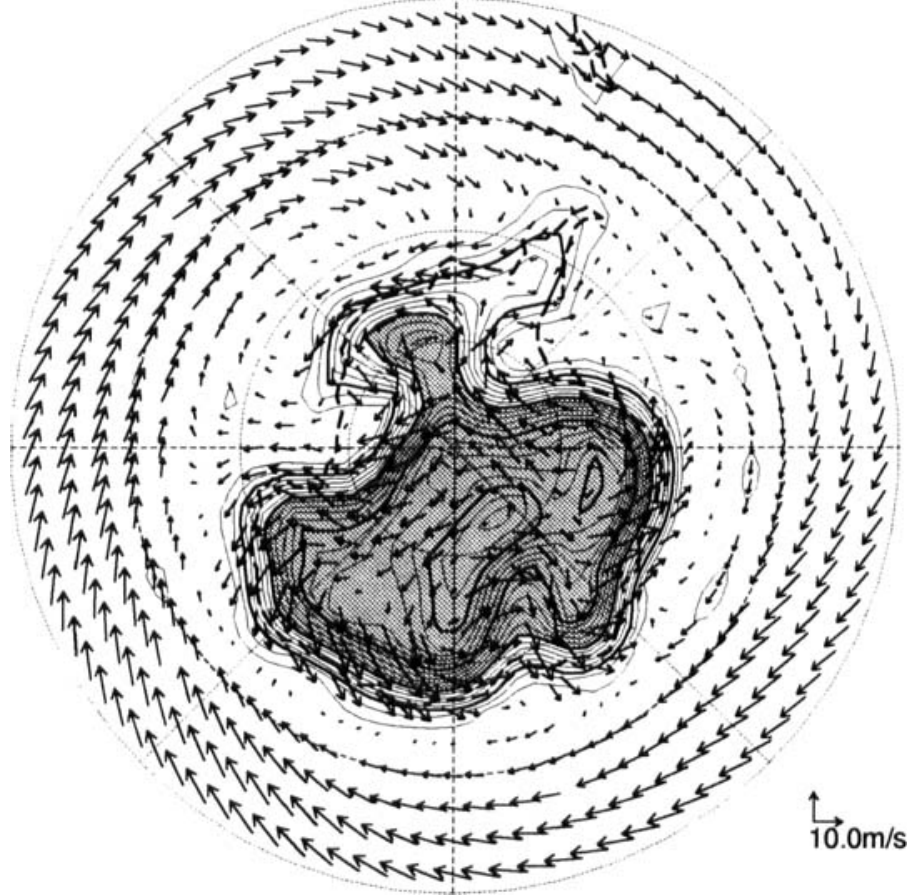

Figure 1. Drainage winds, averaged over June, July and August, on lowest model level, $A=0, B=0.9961$ (see text), about $30 \mathrm{~m}$ above the surface. Polar stereographic projection, extending to $50^{\circ} \mathrm{S}$. The grid is marked at intervals of $10^{\circ}$ latitude and $45^{\circ}$ longitude. The true coastline is marked by a dashed line. Contours show the orography at $200 \mathrm{~m}$ intervals, starting from $100 \mathrm{~m}$, orography higher than $1500 \mathrm{~m}$ is shaded. The velocities around the edge of east Antarctica reach $16 \mathrm{~m} \mathrm{~s}^{-1}$. 

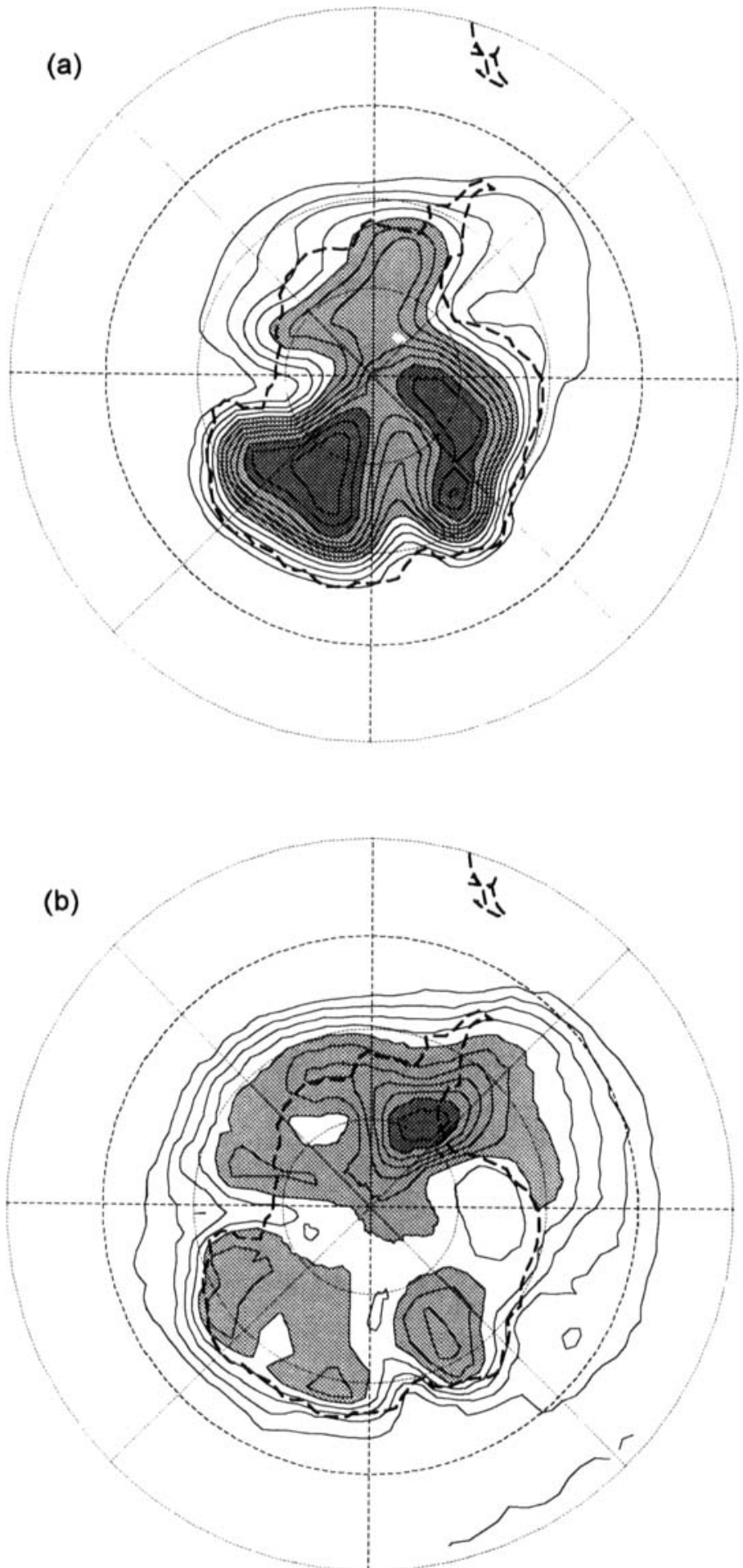

Figure 2. As Fig. 1, but showing the temperature inversion; (a) strength, contour interval $2 \mathrm{~K}$, shading for values $>20 \mathrm{~K}$ (heavy) and $>10 \mathrm{~K}$ (light); (b) mean depth, contour interval $100 \mathrm{~m}$, shading for values $>1000 \mathrm{~m}$ (heavy) and $>500 \mathrm{~m}$ (light). The values are obtained by averaging over the periods when the inversion is present. Inversion frequency is $100 \%$ over the plateau, falling off away from the coast. 
at each grid point are taken over days when an inversion is present at that grid point. For points over the antarctic plateau this includes the entire data record. The June, July, August average inversion strength on the plateau has a maximum value of $25 \mathrm{~K}$, with an average depth of $500 \mathrm{~m}$. Deeper inversions are found to the east of high mountains, suggesting that air in the boundary layer is piling up against the orography. Figure 3 shows the mean winds at the $A=0.3970 \mathrm{kPa}, B=0.9510$ level, about $500 \mathrm{~m}$ above the surface. Around most of the continent the winds at this level are easterly, but where these easterlies encounter strong orographic gradients they are deflected northwards. This is particularly marked in the region of the Lambert glacier, at $60^{\circ} \mathrm{E}$, where a jet extends away from the continent. The western edges of the Weddell and Ross seas are also sites of relatively deep southerly flows; here there is a tendency for the flow to be aligned more nearly parallel to the orographic contours throughout the lower levels, as in a boundary current. As a result the net mass flux from the continent is much larger in these areas, even though the surface winds appear to be fairly uniform around the continent. This suggests that the mesoscale orographic features around Antarctica play an important role in determining the net mass flux.

The model mean sea level pressure, $P_{\text {mal }}$, Fig. 4, shows a strong circumpolar trough, broadly consistent with the observed climatology. Under the high orography of Antarctica, $\boldsymbol{P}_{\mathrm{msl}}$ is sensitive to the method used to extrapolate from surface pressure. The very cold surface temperatures that occur under the inversion are not representative of the bulk of the atmosphere. Hence using these temperatures to generate a bogus temperature profile between the ice surface and mean sea level gives a very high $\boldsymbol{P}_{\mathrm{msl}}$ under the plateau. As a result the gradients associated with the orography dwarf any dynamical variations. For Fig. 4 the bogus temperature profile was based on the temperature at a model level $(A=1.685 \mathrm{kPa}, B=0.8922)$ just above the typical inversion

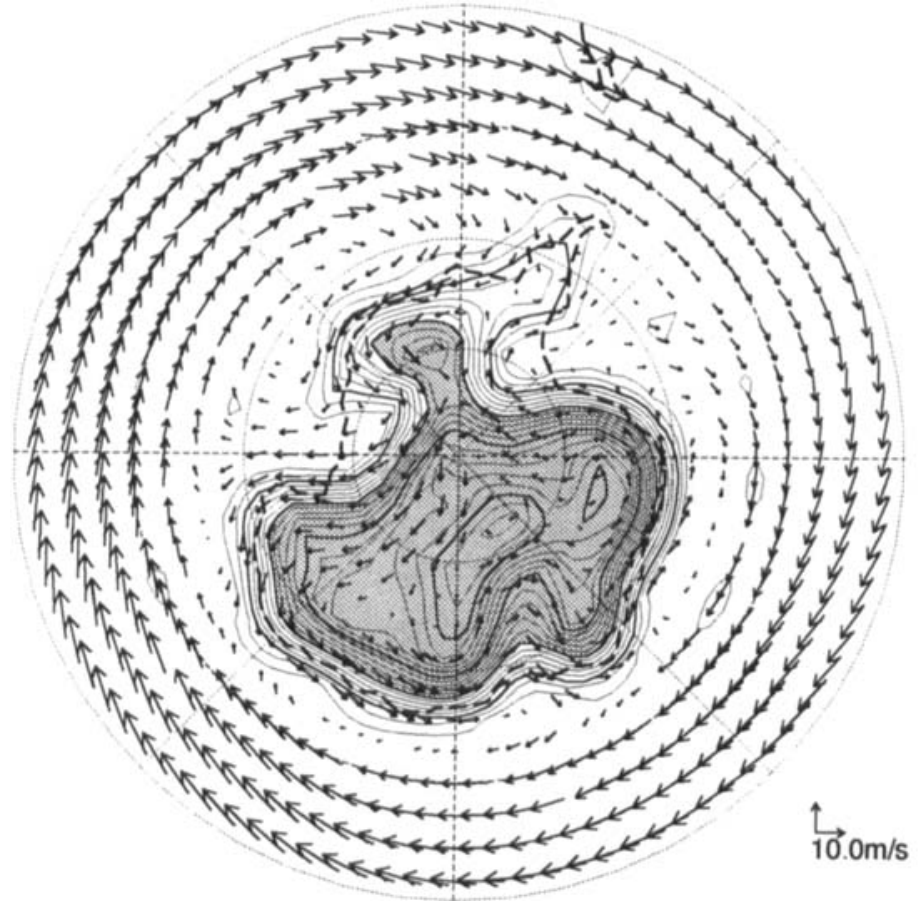

Figure 3. As Fig. 1, but at $A=0.397 \mathrm{kPa}, B=0.9510$. This level is about $500 \mathrm{~m}$ above the surface. 


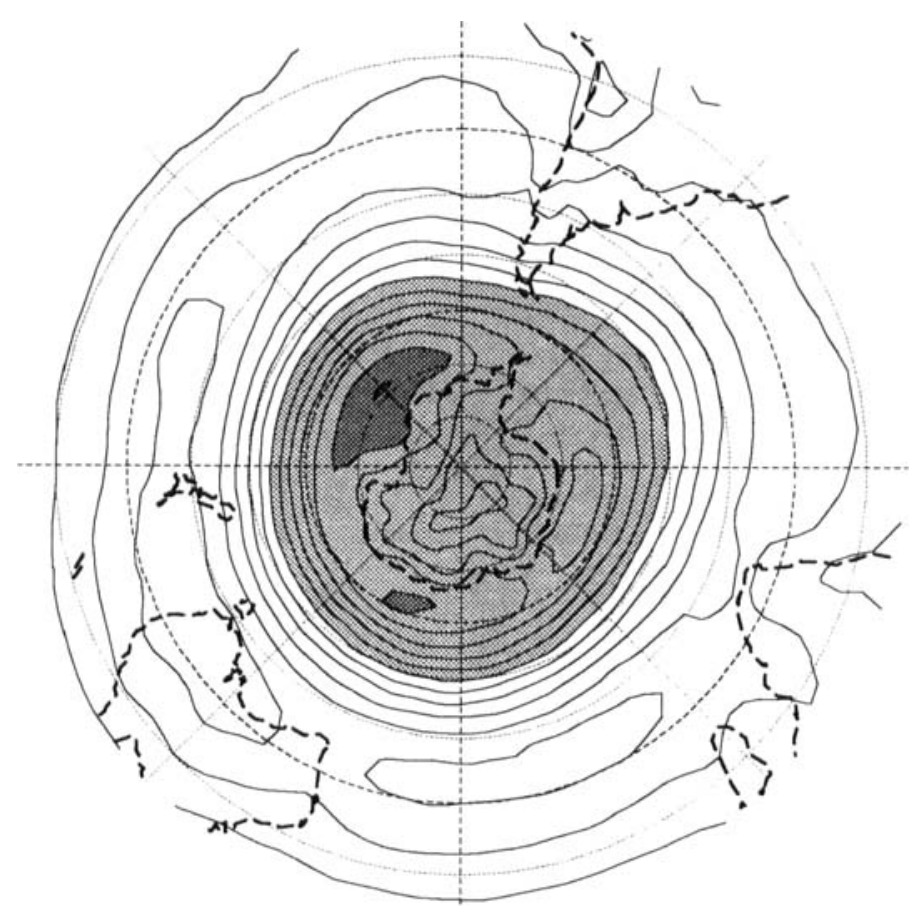

Figure 4. Mean-sea-level pressure (kPa), see text for method of extrapolation to mean sea level. Polar stereographic projection, extending to $15^{\circ} \mathrm{S}$. Contour interval $0.5 \mathrm{kPa}$, shading $<97.5 \mathrm{kPa}$ (heavy) and $<100 \mathrm{kPa}$ (light).

height, using a constant lapse rate of $6.5 \mathrm{~K} \mathrm{~km}^{-1}$. This method produces more meaningful patterns, in which the gradients reflect dynamical activity rather than orography and inversion strength. The model circumpolar trough is deeper than observations, possibly reflecting the poleward displacement of the model jet structure relative to the observations. The wave 3 pattern agrees well with climatology. The latter feature is discussed in more detail by James (1993).

The zonal-mean jet structure is shown in Fig. 5. Before discussing the flow we will describe the format of this and following latitude- $\theta$ figures. The stretching applied to the vertical axes is described in the previous section (Eq. (17)). This has the effect of making the zonal mean pressure, on levels which do not intersect the ground, increase almost linearly with height. In this and all the cross-sections that follow there are five dashed lines plotted for reference. The lower three are, from the bottom up, the minimum, average and maximum surface potential temperature. At $25^{\circ} \mathrm{S}$ and $30^{\circ} \mathrm{N}$ the differences between the maxima and minima reflect the high orography found at those latitudes. At $60^{\circ} \mathrm{S}$ there is no orography or land, so the range of $\theta$ is dominated by the variations of sea-level temperature associated with synoptic waves. Hoskins (1991), extending the terminology of Shaw (1930), introduced the terms overworld, for isentropic surfaces entirely in the stratosphere, underworld, for surfaces which intersect the ground, and middleworld, for those surfaces in between. Since much of the theory discussed later is based on zonal averaging, it is useful to introduce similar terms defined with respect to each latitude. Thus a surface will be said to be grounded if, at a given latitude, it touches the earth's surface, and free if it does not. Iwasaki (1990) has shown that in linear Eady waves the equatorward mass flux takes place entirely on grounded isentropes (i.e. below the maximum surface $\theta$ ). 


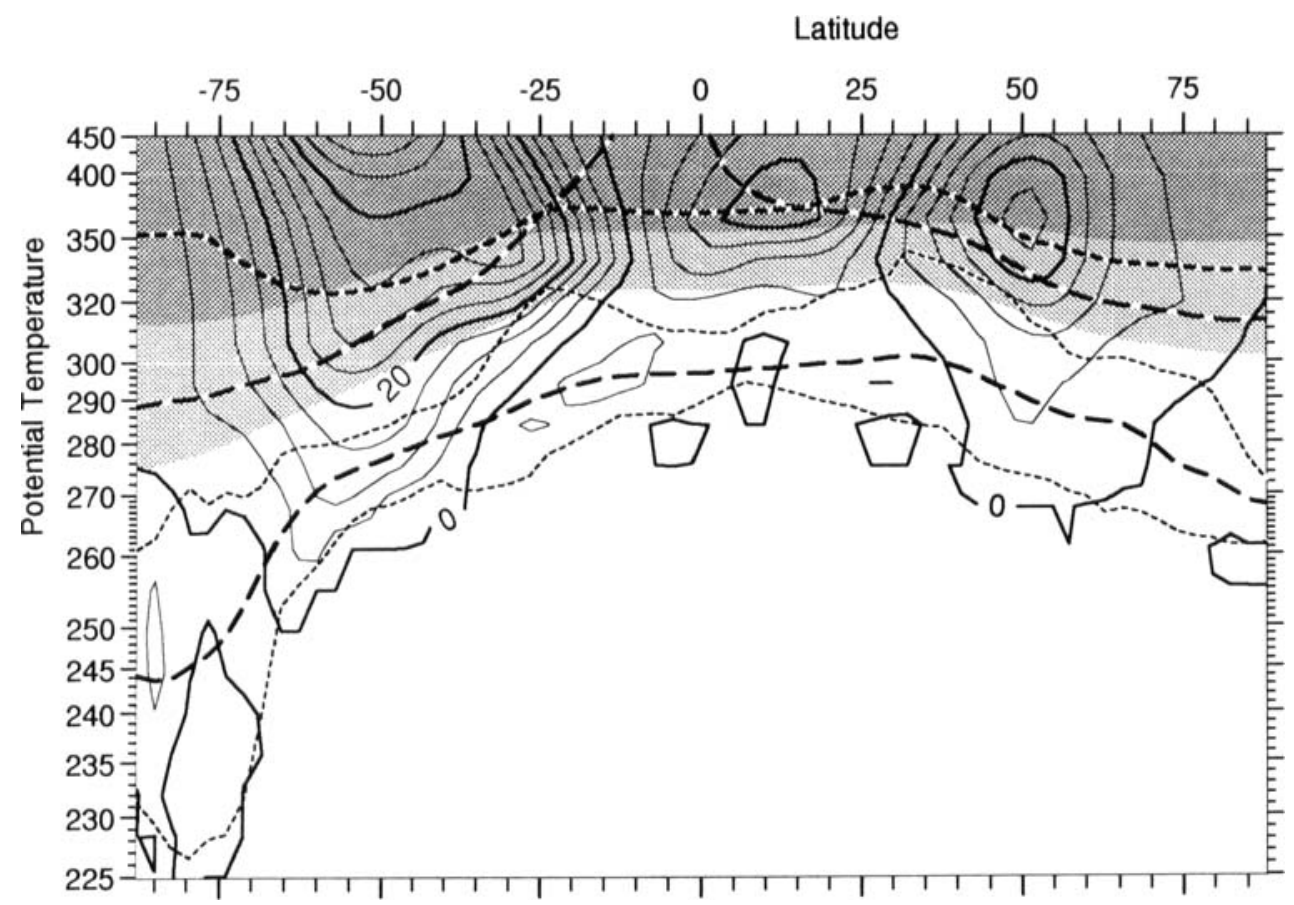

Figure 5. Mass weighted zonal-mean zonal wind $\hat{u}$, contour interval $5 \mathrm{~m} \mathrm{~s}^{-1}$. Shading shows pressure $<20 \mathrm{kPa}$ (heavy) and $<50 \mathrm{kPa}$ (light). See text for an explanation of the dashed lines.

The upper two dashed lines in Fig. 5 show the mean potential temperature at the level of two commonly used indicators of the position of the tropopause; namely the level at which the lapse rate decreases to below $2 \mathrm{~K} \mathrm{~km}^{*}$ (shorter dashes) and the Ertel potential vorticity (PV) $= \pm 2 \times 10^{-6} \mathrm{~K} \mathrm{~m}^{2} \mathrm{~kg}^{-1} \mathrm{~s}^{-1}$ surface (long dashes). The latter provides an indication of the height of the troposphere in the extratropics, since the PV is typically less, in absolute value, than $10^{-6} \mathrm{~K} \mathrm{~m}^{2} \mathrm{~kg}^{-1} \mathrm{~s}^{-1}$ in the troposphere and increases rapidly above the tropopause (e.g. Hoskins et al. 1985). One advantage of using PV to define the tropopause is that it is a material surface for time-scales less than about 5 days, and so can be used to delimit air masses. In this GCM the two measures of the tropopause have very different latitudinal variations. The PV tropopause starts high in the tropics and descends at high latitudes. The meridional circulation (Fig. 6) suggests that the lowest of the two levels at any latitude might be a good indicator of the top of the troposphere. This is consistent with the idea that mid-latitude dynamics are dominated

\footnotetext{
* Because of an error in the radiation code the version of the model used here had a very noisy temperature profile with no unique level satisfying the above condition. To make an objective choice all levels satisfying the lapse-rate condition were assigned a score based on the temperature, moisture and height of the level. The level with the lowest score was then used, corresponding to a cold, dry level near $10 \mathrm{~km}$. The score is given by

$\mathscr{S}=5 \times \max (0,5-z)+5 \times \max (0, z-12)+2 \times\left(\mathcal{T}-\mathcal{T}_{\min }\right)+2 \times \max \left(0, T_{L-1}-T_{L-2}\right)+C_{Q}+C_{\mathrm{b}]}$

where $\mathscr{J}=T+2 z$. The first two terms score against very high or low levels. The third term favours the coldest point relative to a lapse rate of $2 \mathrm{~K} \mathrm{~km}^{-1}$. The fourth scores against the upper inversion when a pair occur separated by a single warm point. $C_{H}=10$ if relative humidity $H<5 \%$ and zero otherwise. $C_{\mathrm{bl}}=50$ if the level is in the bottom three levels, zero otherwise. This last factor eliminates surface inversions. $z$ is the height in $\mathrm{km}$ and $L$ is the model level number.
} 

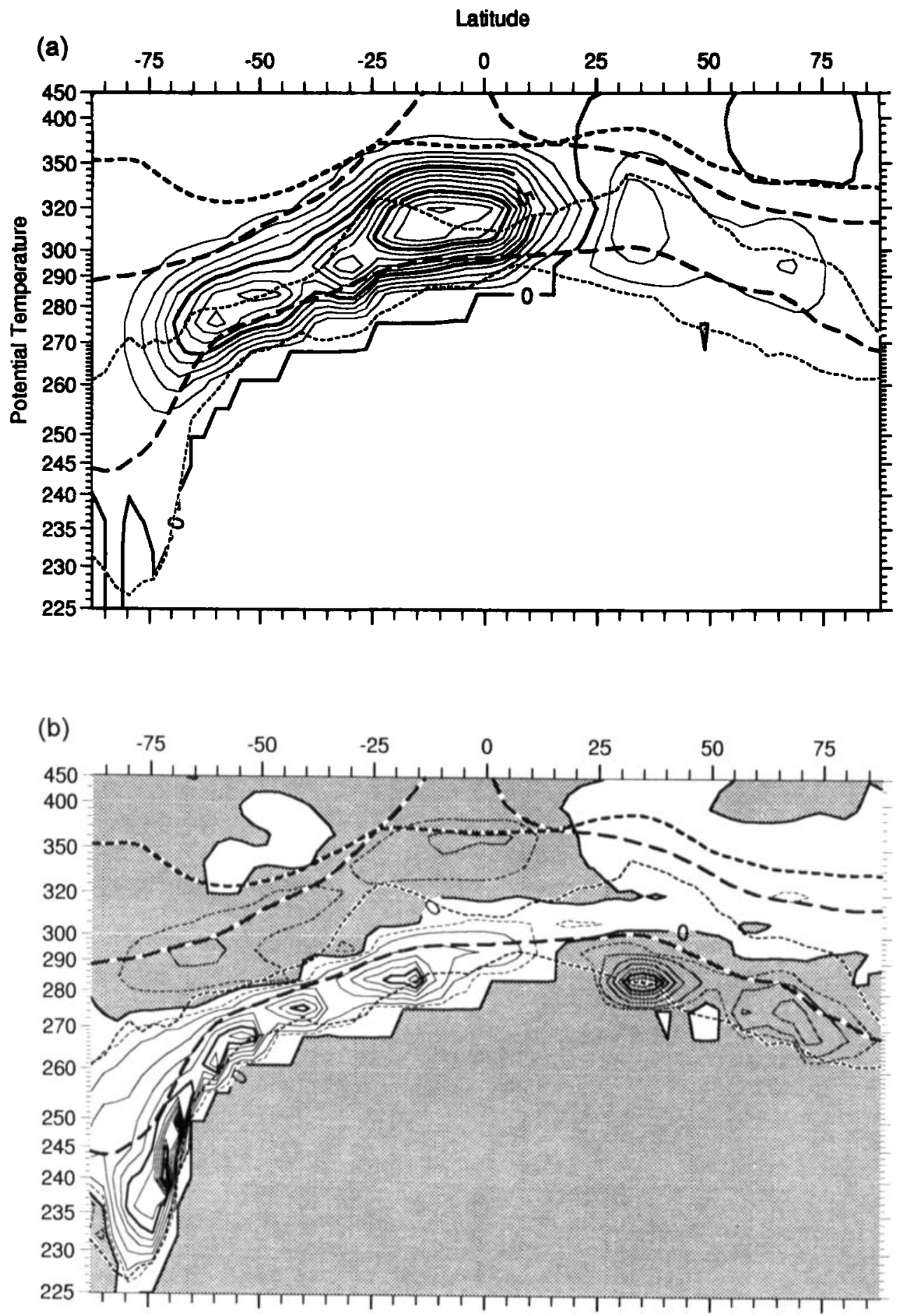

Figure 6. As Fig. 5, but showing: (a) mean meridional mass stream function, contour interval $10^{10} \mathrm{~kg} \mathrm{~s}^{-1}$; (b) mass-weighted mean meridional velocity, negative values shaded, solid contours every $1 \mathrm{~m} \mathrm{~s}^{-1}$ and dashed contours at $\pm 0.5 \mathrm{~m} \mathrm{~s}^{-1}$. 
by the properties of potential vorticity, whilst in the tropics the temperature structure, and the associated convective activity, is more important.

We now return to the zonal-mean jet structure shown by the contours in Fig. 5 . The model has a hint of a double jet structure in the southern hemisphere, but there is no distinct velocity maximum. The jets are slightly poleward of their climatological positions. Climatologies (Hoskins et al. 1983; Randel 1987) show a distinct maximum at $30^{\circ} \mathrm{S}, 20$ $30 \mathrm{kPa}$. The model agrees reasonably well with the observations in putting the maximum in the vertical shear of the low-level wind at $50-60^{\circ} \mathrm{S}$.

The meridional circulation is shown in Fig. 6, the mass stream function in Fig. 6(a) and the mass-weighted mean meridional velocity in Fig. 6(b). The stream function shows an extended Hadley cell, similar to that in the First GARP* Global Experiment (FGGE) observations analysed by Johnson (1989). Experiments with the UGCM have shown that the strength of the Hadley cell in the tropics is sensitive to the form of radiation parametrization used (Slingo et al. 1991). The extratropical mean meridional circulation is robust with respect to such changes. Maxima in upper tropospheric $\hat{v}$, of about $1 \mathrm{~m} \mathrm{~s}^{-1}$, occur in the tropics and at the poleward flank of the winter storm track. Large mean meridional velocities occur at the lower boundary, with $\hat{v}$ values of up to $11 \mathrm{~m} \mathrm{~s}^{-1}$. These values occur below the average surface potential temperature, and are associated with cold polar air moving equatorwards (contours of the stream function appear below the minimum surface potential temperature because of the spreading that occurs first when the stream function is interpolated from half to full $\theta$-levels and again in the process of contouring). In the Lorenz formulation the mean density decreases below max $\left(\theta_{\mathrm{s}}\right)$. This is because air at these levels occupies a decreasing fraction of a latitude circle as $\theta$ is reduced, so $\int \sigma d \lambda \rightarrow 0$ as $\theta \rightarrow \min \left(\theta_{s}\right)$. In these results the lower, equatorwards branch of the circulation is at lower density than the upper branch. The pressure difference, $\Delta p$, across the lower branch is also smaller than that across the upper branch. Since the mass flux $(\approx \hat{v} \Delta p)$ is equal and opposite in the two branches the velocities are larger in the lower branch. The circulation transports energy polewards, since air in the upper branch has higher static energy, $e=\Phi+c_{p} T+\mathscr{L} q$, than air in the lower branch $(\mathscr{L}$ is the latent heat of vaporization and $q$ the water mixing ratio). A similar circulation occurs in the ocean (Bryan 1987). The high-internal-energy fluid moving polewards at the surface of the ocean loses heat to the low-energy fluid moving equatorwards in the atmosphere.

Iwasaki (1992) has carried out a similar analysis on the NCAR Community Climate Model 1. In the stream function he finds a clear split between the Hadley cell in the tropics and an extratropical cell, which is unlike the single unbroken cell found here. The similarity between the present results and the FGGE analysis of Johnson (1989) is most likely due to the similarity between the model used here and that used to assimilate the FGGE data. The vertical velocity is not measured directly so the meridional circulation is sensitive to the first guess provided by the model in the assimilation process. Preliminary analysis of more recent results from the UGCM, using an improved convection scheme, shows a greater degree of separation between the two regions.

The isentropic mean is sometimes referred to as being 'quasi-Lagrangian' because the averaging volume follows the vertical component of the parcel motion to some extent. Different variants on this theme are possible. Vertical velocities become small near the surface, so averaging at constant height and using potential temperature as a meridional coordinate is a useful option. It can be shown (Hoskins 1991) that the mean mass flux is in this case polewards at the lower boundary.

* GARP = Global Atmospheric Research Program-a joint World Meteorological Organization/International Council of Scientific Unions project. 
The vertical motion associated with these meridional velocities has a maximum cooling of $3.5 \mathrm{~K} \mathrm{day}^{-1}$ near the surface at the pole, and a maximum heating of $7 \mathrm{~K} \mathrm{day}^{-1}$ at the surface where cold air flows off the antarctic continent over the relatively warm oceans.

The meridional circulation produces a torque on the zonal flow; see Fig. 7. The maximum values occur over the edge of Antarctica, up to $15 \mathrm{~m} \mathrm{~s}^{-1}$ day ${ }^{-1}$. This is the mean taken over a season in which the net change in the zonal wind is only a few $\mathrm{m} \mathrm{s}^{-1}$, so this forcing must be balanced by other forcing terms. It is shown later that the EPflux convergence balances this forcing, the dominant contribution coming from the vertical component.

Another view of the mean circulation is given in Fig. 8. This shows a pressurelongitude section around $75^{\circ} \mathrm{S}$. The wind barbs show the seasonal mean horizontal winds averaged on model surfaces. The lower potential-temperature contours follow the topography of Antarctica to a surprising extent. The strong cooling on the high plateau creates an unusual situation in which the potential temperature at the top of the mountain is lower than that at the base. The drainage flow brings potentially cold air down, which is then warmed radiatively. It is not clear how far this behaviour is realistic.

\section{TRANSIENT AND EDDY FLUXES}

Before going into a detailed breakdown of the momentum budget we will look at the synoptic distribution of the transient eddies (Fig. 9). Following the example of Blackmon et al. (1977), Hoskins et al. (1983) and others, the eddies are divided into high- and low-frequency components. This division is intended to separate the higherfrequency synoptic eddies, which get most of their energy from baroclinic instability,

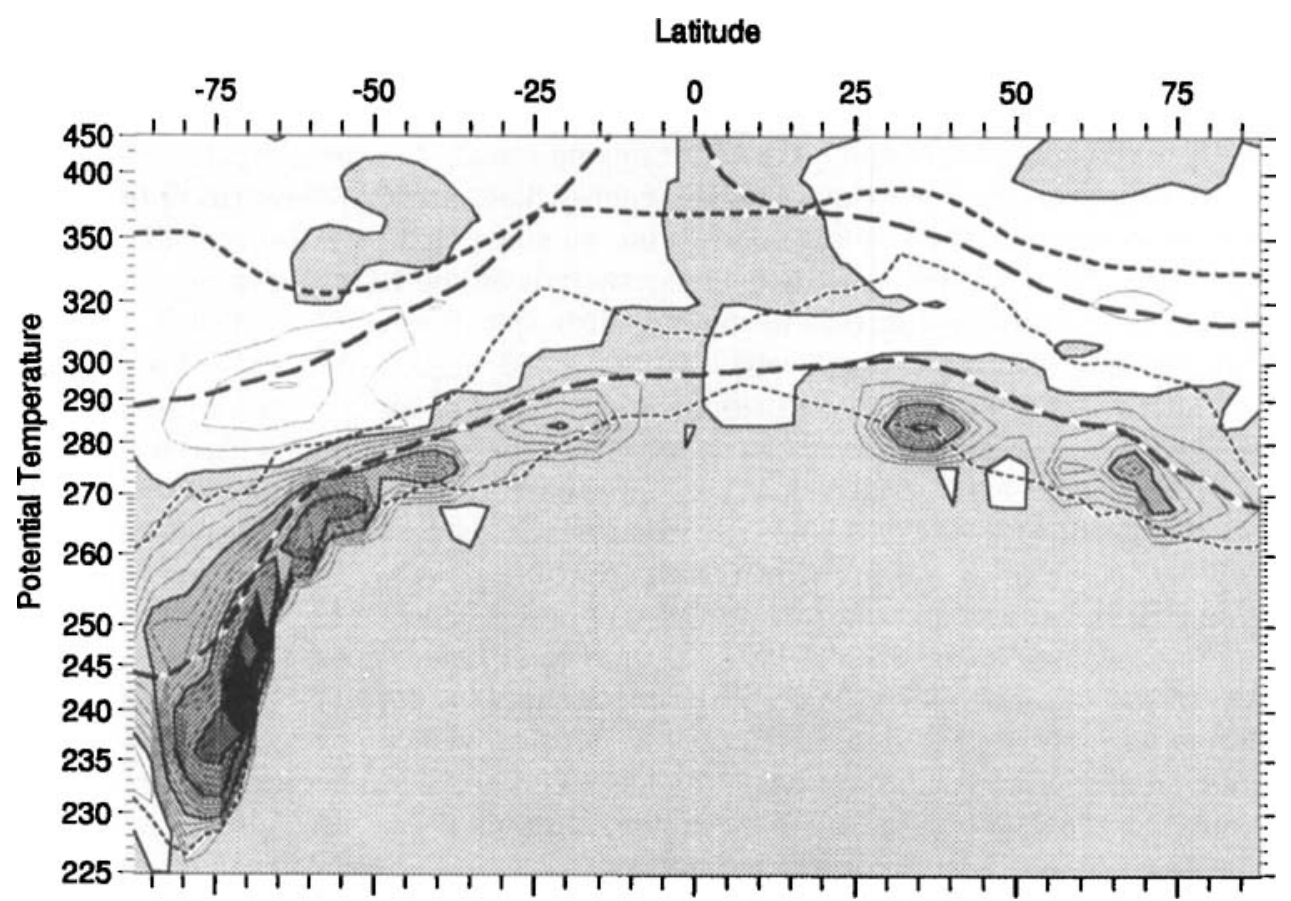

Figure 7. As Fig. 5, but showing the forcing of the zonal-mean zonal flow by the meridional circulation, of $f-(1 / a \cos \phi) \partial(\hat{a} \cos \phi) / \partial \phi)$. Contour interval $5 \mathrm{~m} \mathrm{~s}^{-1}$ day ${ }^{-1}$, negative values shaded. 


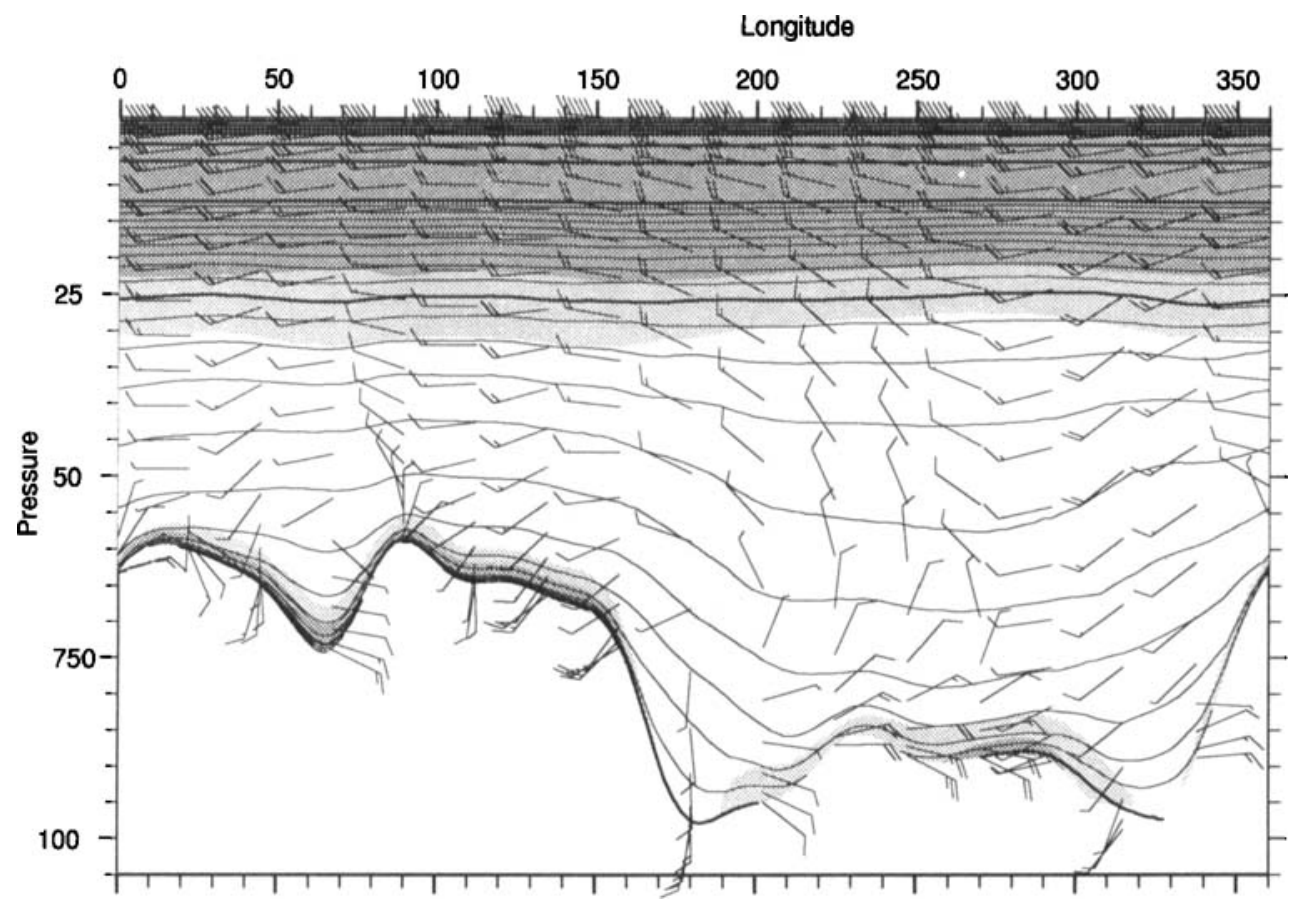

Figure 8. Pressure-longitude section at $75^{\circ} \mathrm{S}$. The lower limit of the contours and wind barbs delimits the orography. The seasonal time average has been taken on model surfaces and plotted as a function of the seasonal mean pressure. This method avoids interpolation to pressure levels which would smear out the finescale structure in the boundary layer. The shading shows potential-vorticity values, in units of $10^{-6} \mathrm{~K} \mathrm{~m}^{2} \mathrm{~kg}^{-1} \mathrm{~s}^{-1}$, $<-4$ (heavy) and $<-2$ (light). High values occur both in the stratosphere and in the strongly stratified inversion layer. Potential temperature is contoured, interval $5 \mathrm{~K}$ up to $350 \mathrm{~K}$, and $50 \mathrm{~K}$ above that. The lowest heavy contour, which lies near the surface of Antarctica, is $250 \mathrm{~K}$. Wind barbs are shown for the horizontal wind, each flight corresponding to $5 \mathrm{~m} \mathrm{~s}^{-1}$.

from the lower-frequency, larger-scale eddies. In practice, the two regimes cannot be separated cleanly, but the filtered fluxes nevertheless give an indication of different patterns of behaviour. Here the high-frequency eddies are defined to be those with periods less than 6 days. Fourier analysis was used, giving a sharp spectral cut-off, with a data record of 96 days. Analysis of the cross-power spectrum has been carried out and shows that most of the variance is associated with structures moving at the speed of the mean flow. This suggests that advection is the principal factor determining the variation of frequency as a function of scale.

The storm track, as defined by the high-frequency temperature flux, is elongated, almost circling the globe. This contrasts with the climatology, which has the storm track breaking up near the longitude of New Zealand (James and Anderson 1984; Trenberth 1991). The high-frequency fluxes show strong meridional gradients, the activity decreasing rapidly polewards of about $60^{\circ} \mathrm{S}$. This could be due simply to the fact that the $75 \mathrm{kPa}$ surface intersects the orography of Antarctica, but similar gradients are seen in the fluxes at $50 \mathrm{kPa}$, suggesting that there is a physically significant change in the distribution of activity. The sharp edge of the high-frequency activity is lost if a filter with a broader frequency cut-off is used. Increasing the resolution to T63 does not significantly alter the distribution of the high-frequency fluxes (not shown).

The small-scale noise in the fluxes is largely caused by temporal aliasing of frequencies not fully resolved by the 18-hourly data. In general nothing precise can be said about 

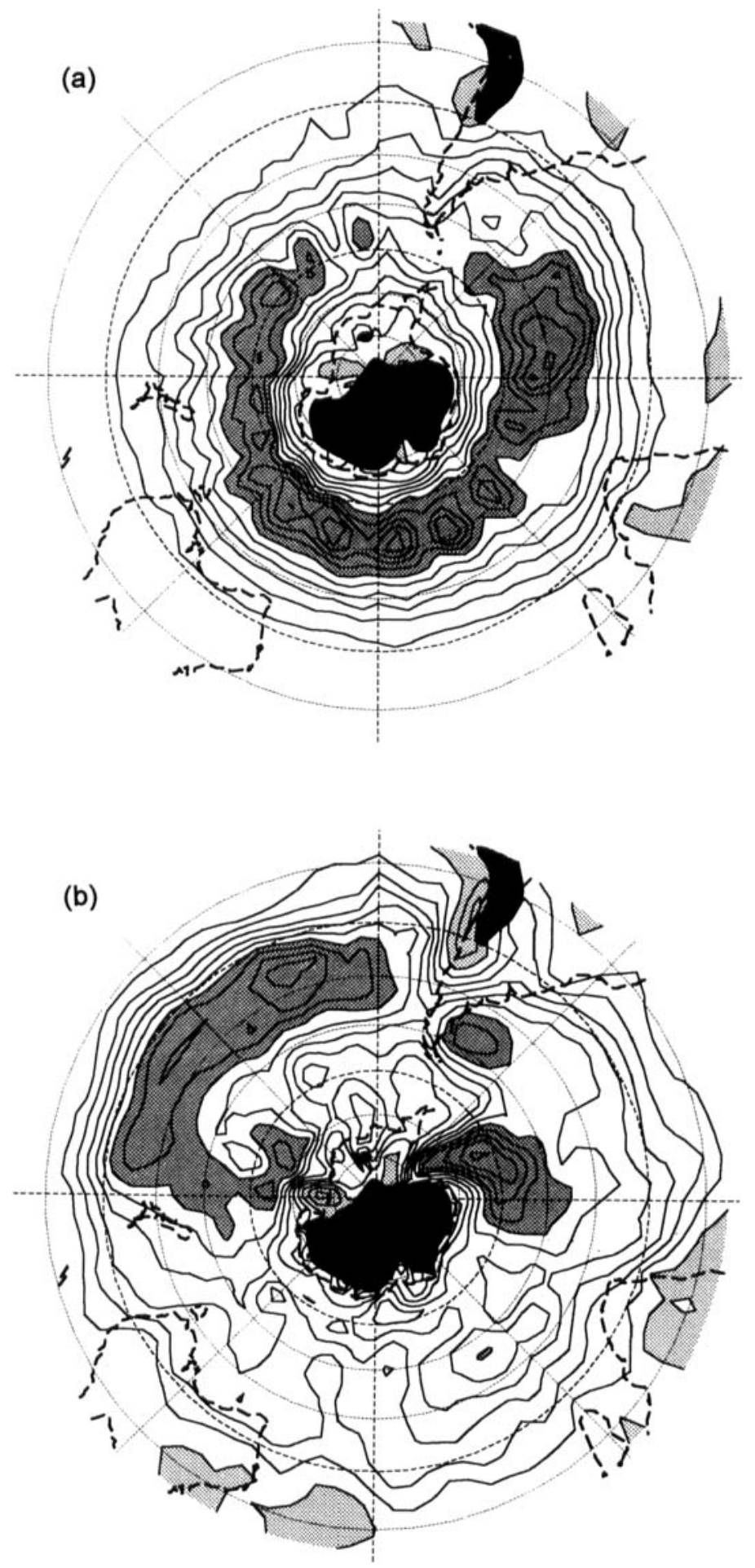

Figure 9. As Fig. 4, but showing the meridional heat flux at $75 \mathrm{kPa}$ in the T42 experiment; (a) high-frequency eddies (periods less than 6 days), (b) low-frequency eddies. Contour interval $2 \mathrm{~K} \mathrm{~m} \mathrm{~s}^{-1}$, shading shows values $<-12 \mathrm{~K} \mathrm{~m}^{-1}$ (heavy) and $>0 \mathrm{Km} \mathrm{s}^{-1}$ (light). Areas with mean surface pressure $<75 \mathrm{kPa}$ are blacked out. 
these unresolved frequencies, but the uniform westerly propagation found in the southern storm track makes a definite statement possible here. Westerly waves with periods less than 36 hours alias onto easterly waves with periods greater than 36 hours. These bogus easterly waves interfere with the resolved westerly waves to produce standing waves which give regularly spaced peaks and nodes in the flux amplitude.

The low-frequency eddy heat fluxes are less localized than the high frequencies. The distribution of synoptic features in the flux, such as the maximum in the Weddell Sea, is not robust. Changing the model resolution and parametrizations redistributes such features. The most important feature, in the context of this study, is that the lowfrequency fluxes extend further polewards than the high-frequency fluxes: this feature is robust. Yasunari and Kodama (1993) have observed a low-frequency oscillation in the troposphere over Antarctica. The structure of the low-frequency variance in the UGCM appears to be qualitatively similar, consisting of a periodic displacement of the polar vortex with a period of a month or so.

The forcing of the mean flow by the eddies can be quantified by using the EP flux, discussed in section 3 . Figure 10 shows the total forcing of the zonal-mean zonal flow. This figure includes transient and stationary waves, the transients dominate. Orographic drag is discussed further later. The distribution of the flux divergence resembles that of $f \hat{v}$, the largest values occur near the lower boundary. The formulation used ensures that the values are well defined, and that no spurious sources are generated by the interpolation. The largest eddy flux divergence is associated with the coldest air, coincident with the greatest equatorwards velocity. This pattern agrees with that found by Iwasaki (1990) in an analysis of the Eady mode of baroclinic instability. The fact that all the divergence occurs on grounded $\theta$-surfaces reflects the importance of surface meridional $\theta$-gradients in the baroclinic instability.

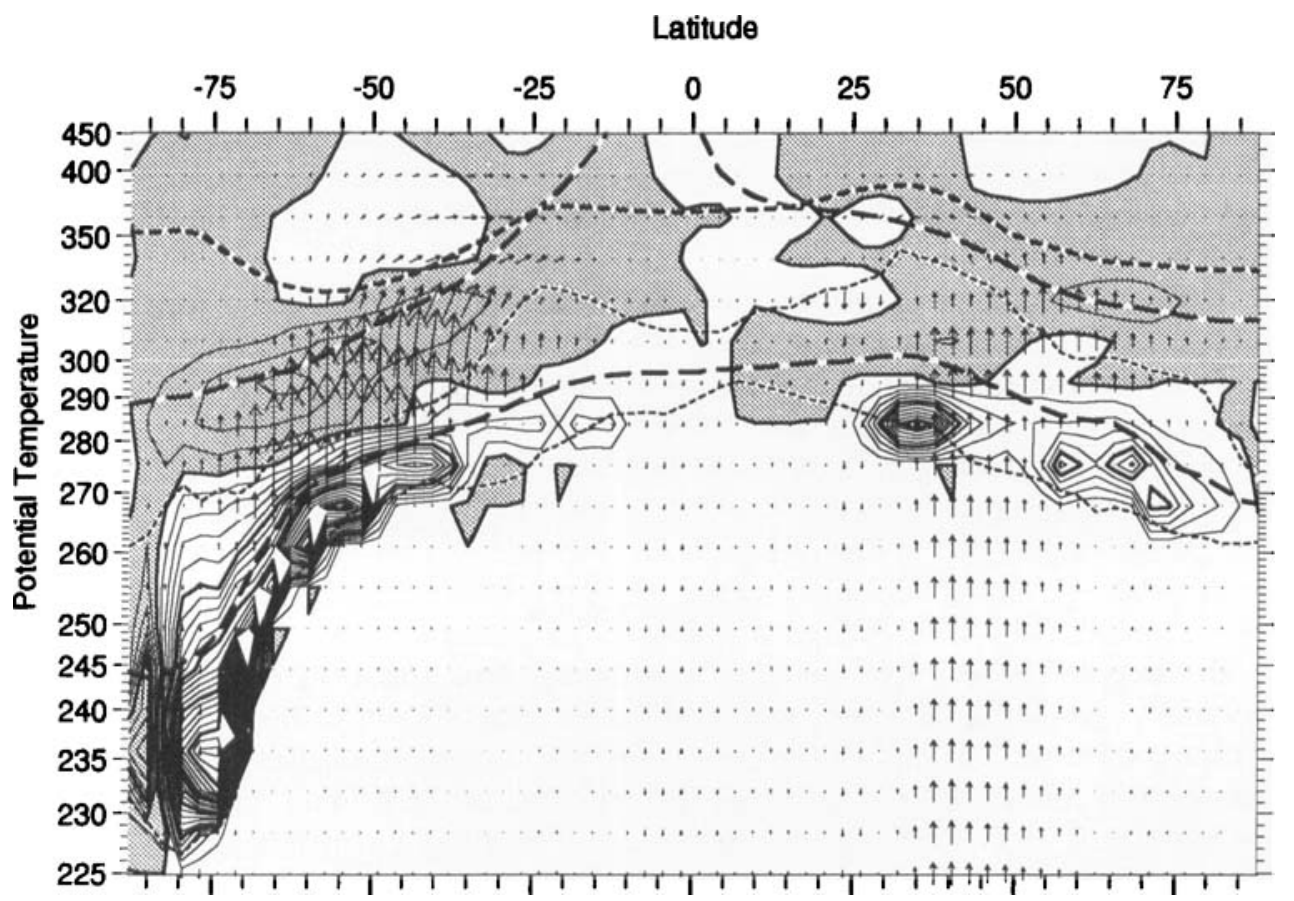

Figure 10. As Fig. 5, but showing the forcing of the zonal-mean zonal flow by the eddies, total EP-flux divergence (contour interval $5 \mathrm{~m} \mathrm{~s}^{-1}$ day $^{-1}$, negative values shaded) and the EP-flux vectors. 
In the upper troposphere $f \hat{v}$ and $\nabla \cdot F$ balance closely, implying that unresolved processes, represented by $\hat{\boldsymbol{X}}$ in Eq. (7), contribute a relative small amount to the momentum budget in this region. Near the surface there is a significant imbalance, of the order of $30 \%$. The parametrized friction is presumably responsible for the difference. Though friction is important in the boundary layer it does not dominate the eddy momentum fluxes in the way assumed by Ball (1960). The large-scale eddy momentum fluxes are, in this experiment, the same order of magnitude as the friction terms in the zonal-mean momentum budget.

Figure 11 shows the contributions of the vertical and horizontal components of the EP flux (Figs. 11(a) and 11(b) respectively). (Note the change in contour interval.) It is clear that the vertical component dominates. As discussed in section 3 , the vertical component of the EP flux is related to temperature fluxes on pressure surfaces. These fluxes are usually associated with developing baroclinic instability. Although the disturbances may be strongly nonlinear, this result suggests that there is significant growth of disturbances in-situ at high latitudes: the momentum fluxes cannot be explained through decaying storms propagating in from the main mid-latitude storm track.

The vectors in Fig. 10 also give an indication of the importance of orographic drag. The vertical component of the EP flux becomes constant under the lowest surface $\theta$ value, taking the value of the zonal-mean orographic drag. The latter is significant near the South Pole and also in the northern hemisphere at the latitude of the Himalayas, but weak elsewhere (note that the amplitude of the arrows in the figure tends to zero at the poles because they represent the net angular momentum flux, which includes a factor of $\cos \phi$ ). The acceleration that these fluxes would cause if they were to propagate vertically and be absorbed into a layer of the atmosphere $20 \mathrm{kPa}$ thick has a peak value of about $12 \mathrm{~m} \mathrm{~s}^{-1}$ day $^{-1}$ at $85^{\circ} \mathrm{S}$. Between 70 and $80^{\circ} \mathrm{S}$ the absolute value of the drag is less than a tenth of this value, and the sign oscillates.

Figure 12 shows the forcing terms in Eq. (13), in which the vertical EP flux and the geostrophic mass flux have been eliminated. Figure 12(a) shows the forcing due to the ageostrophic circulation, negative values (light shading) correspond to equatorward motion. In mid latitudes there is a large thermally indirect circulation, the Ferrel cell, stretching from $65^{\circ} \mathrm{S}$ to $30^{\circ} \mathrm{S}$. In the tropics and high latitudes the circulation is thermally direct. Figure 12(b) shows the advection of relative vorticity (last term on the left-hand side of Eq. (13)).

In the upper polar troposphere the main balance is between the horizontal EP-flux divergence (Fig. 11(b)) and the ageostrophic circulation. The forcing due to the lower branch of the ageostrophic circulation must be balanced by friction. In the tropics the advection of relative vorticity is more important. The residual also has a greater relative magnitude in the tropics, reflecting the greater importance of vertical advection and other parametrized processes $(\hat{X})$.

\section{Discussion}

The accepted conceptual model for the drainage flow, following Ball (1960), is based on a zonally symmetric flow, with meridional advection of absolute momentum balanced by vertical diffusion. In this GCM experiment the dominant balance is between mean advection and convergence of the vertical component of the EP flux. The importance of eddy mass flux in the meridional circulation is well known in connection with mid latitudes. There the Eulerian mean circulation is thermally indirect, rising poleward of the jet and sinking on the equatorward side. When the eddy mass flux is taken into consideration, the net mass flux is in a thermally direct sense, with warm air rising in the 

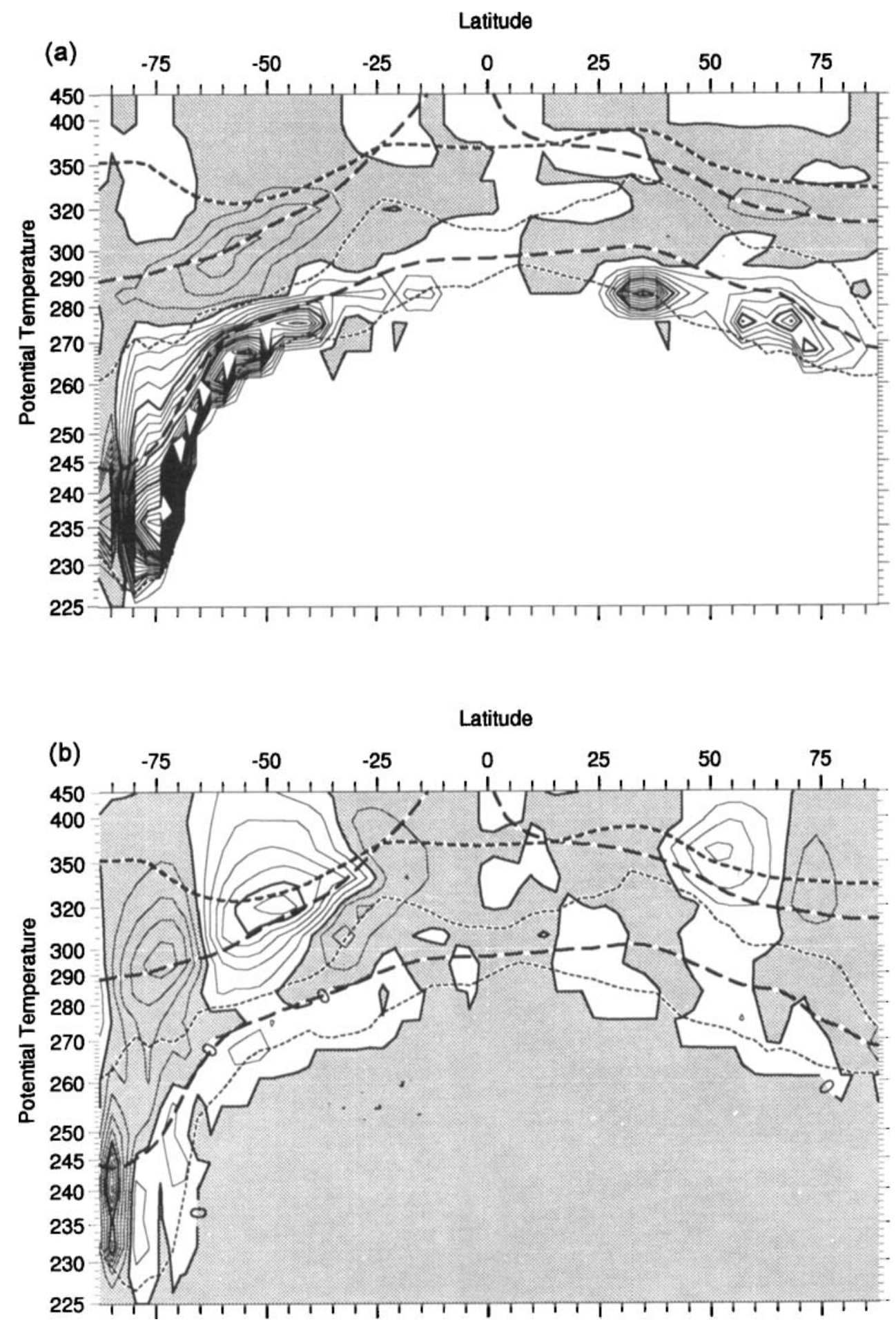

Figure 11. As Fig. 5, but showing the two components of the eddy forcing: (a) divergence of the vertical EP flux, contour interval $5 \mathrm{~m} \mathrm{~s}^{-1}$ day $^{-1}$; (b) divergence of the horizontal EP flux, contour interval $1 \mathrm{~m} \mathrm{~s}^{-1} \mathrm{day}^{-1}$. Negative values are shaded in both cases. 

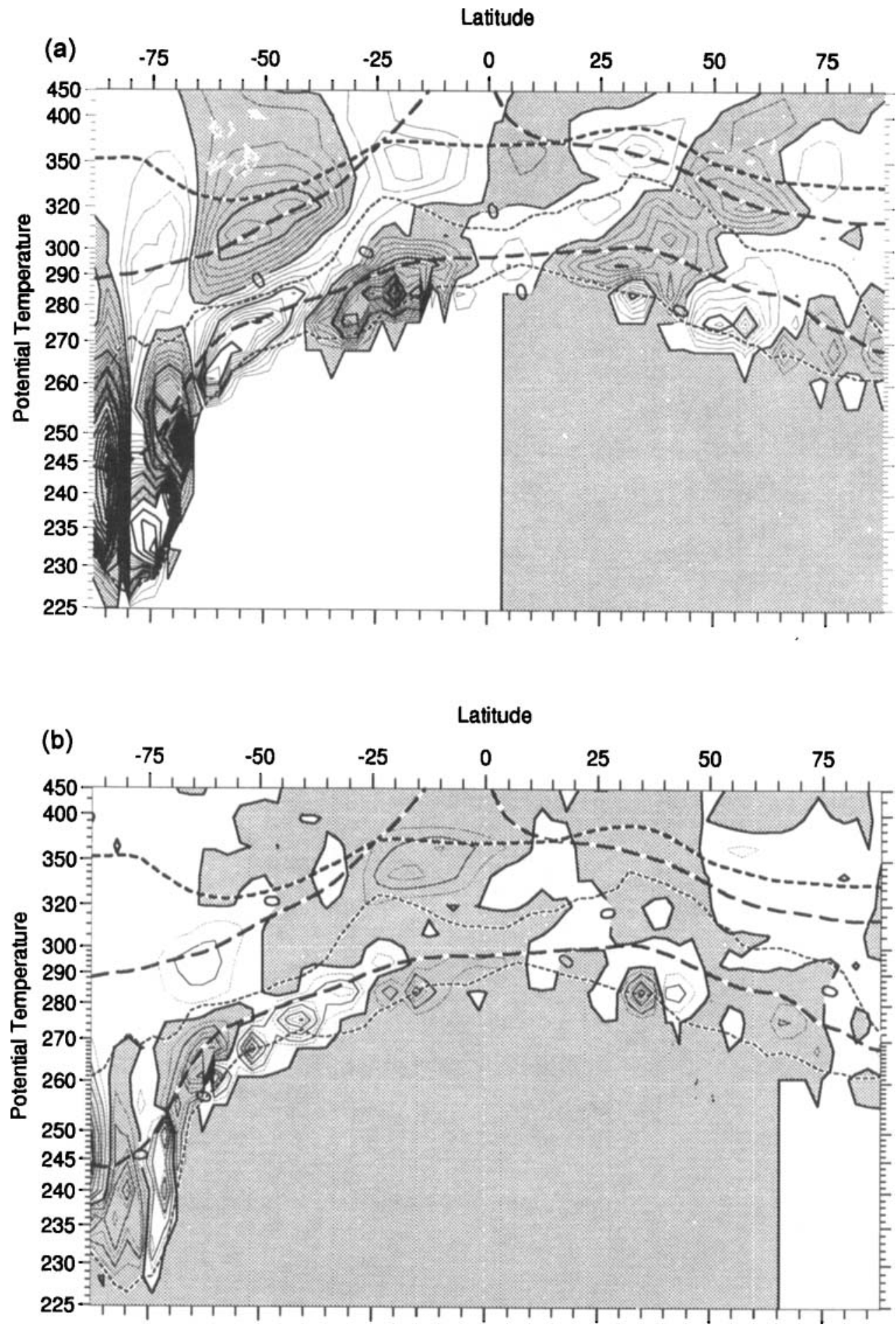

Figure 12. The main terms in the transformed momentum equations: (a) forcing by the ageostrophic circulation, $-f \hat{v}_{\mathrm{ag}}$, contour interval $1 \mathrm{~m} \mathrm{~s}^{-1}$ day ${ }^{-1}$; (b) flux of relative vorticity by mean meridional velocity, contour interval $0.5 \mathrm{~m} \mathrm{~s}^{-1}$ day ${ }^{-1}$ with solid contours every $1 \mathrm{~m} \mathrm{~s}^{-1}$ day $^{-1}$. Negative values shaded in both cases. 
tropics, as shown in Fig. 6(a). The new result is the importance of the eddy mass flux over Antarctica and in the drainage flow. More research needs to be done into the structures of the systems responsible for these stresses.

Although Eq. (13) provides a useful decomposition of the momentum budget there are a couple of problems with the physical interpretation of $\hat{v}_{\text {ag. }}$. Firstly, $f v_{\text {ag }}$ is calculated as $f v-\rho^{-1} \partial p / \partial \lambda$, so does not necessarily vanish at the equator. Secondly, while the vertical integral of $\hat{v}$ vanishes in a sufficiently long time mean the same is not true of $\hat{v}_{\mathrm{g}}$ and $\hat{v}_{\mathrm{ag}}$ independently. The vertical integral of the former is in fact the zonal-mean orographic form drag. As mentioned earlier, this is small at most latitudes but significant near the southern pole. This is reflected in $\hat{v}_{\text {ag }}$ being positive through virtually the entire model depth at the South Pole.

Recent observational work has shown that the zonal-mean eddy fluxes in the southern storm track undergo a systematic oscillation, with a period near 14 days (Randel and Stanford 1985). The same type of behaviour is found in this model. Figure 13 shows a time series of the vertical EP-flux divergence at $322 \mathrm{~K}$. At high southern latitudes the time variation is dominated by periods of the order of 2 weeks. The 14-day fluctuations in the storm track are unexpected, given that this is the zonal mean and the storms moving around the southern hemisphere have a superficial appearance of propagating
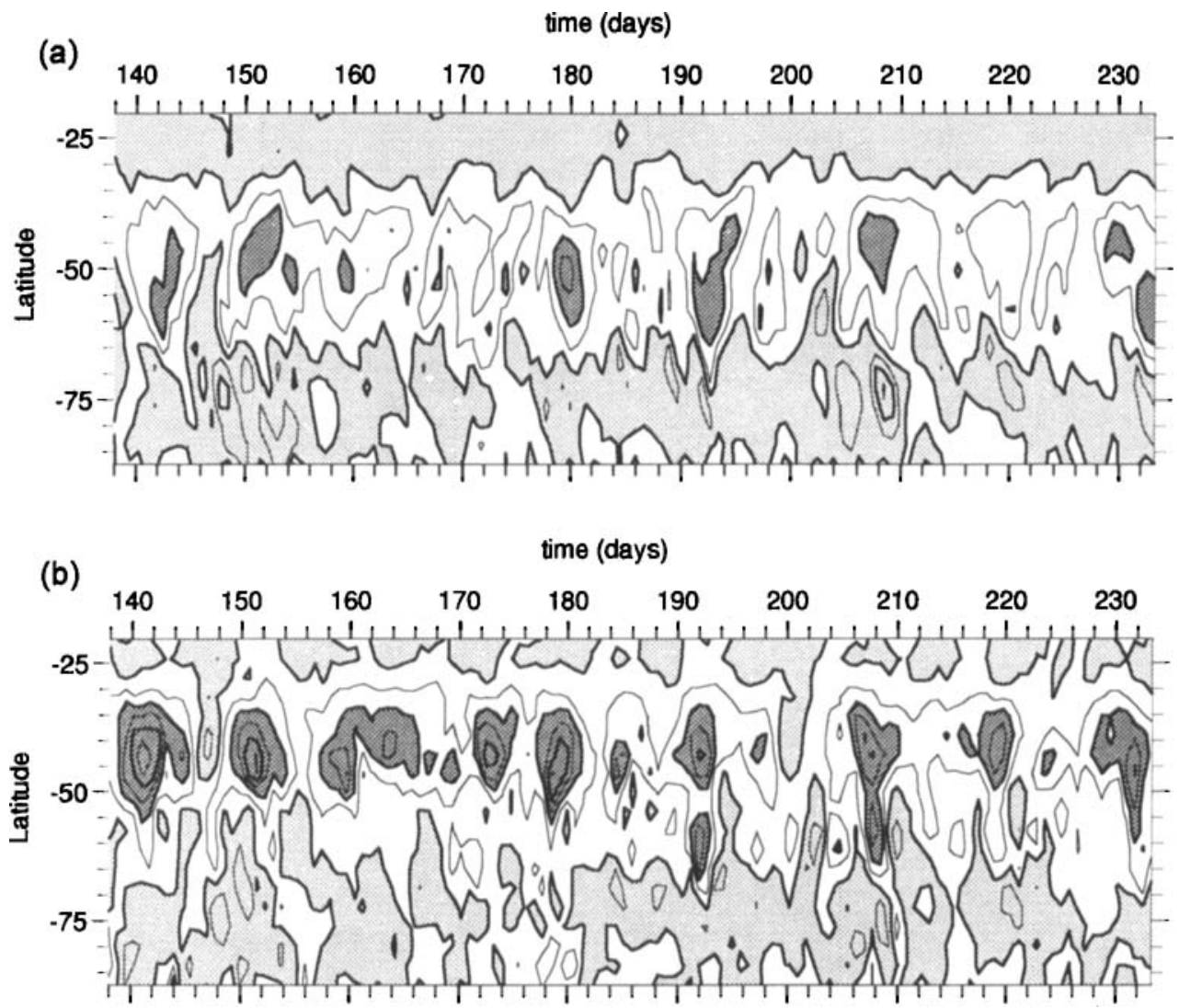

Figure 13. Time/latitude plot of eddy forcing of the zonal-mean zonal wind on the $\theta=322 \mathrm{~K}$ surface, from 20 to $88^{\circ} \mathrm{S}$ and for days 138 to 232.5 , i.e. June, July and August. (a) Horizontal EP-flux divergence, shading $>10 \mathrm{~m} \mathrm{~s}^{-1}$ day $^{-1}$ (heavy) and $<0$ (light), (b) vertical EP-flux divergence, shading $<-10 \mathrm{~m} \mathrm{~s}^{-1}$ day $^{-1}$ (heavy) and $>0$ (light). Contour interval $5 \mathrm{~m} \mathrm{~s}^{-1}$ day $^{-1}$. 
with a near-steady amplitude. Similar behaviour has been seen in a baroclinic life-cycle simulation (Barnes and Young 1992).

The changes in the zonal-mean zonal wind (Fig. 14) correlate better with the meridional momentum flux than with the vertical momentum flux, even though the vertical flux is much stronger. At $50^{\circ} \mathrm{S}$ the acceleration of the zonal-mean wind has a correlation of 0.76 with the horizontal EP-flux convergence. The significance of this correlation can be estimated using Student's $t$-test, but the answer depends on the number of degrees of freedom $(n)$ attributed to the times series. The significance values for $n=$ 10 and 20 are $99 \%$ and $99.9 \%$ respectively. The acceleration of the zonal-mean wind has a correlation of -0.11 with the vertical EP-flux convergence, which is not significant. A significant correlation of -0.53 was found between the mid-level $(302 \mathrm{~K})$ wind and the lower-level ( $286 \mathrm{~K}$ ) EP flux (significance $88 \%$ and $98 \%$ at $n=10$ and 20 respectively). A similar oscillation was found by Pfeffer (1992). The vertical flux is balanced primarily by meridional motions, but because the horizontal fux has a non-zero barotropic component it cannot be cancelled to the same degree. This contrasts with the situation in the stratosphere (Edmon et al. 1980) where total EP flux divergence tends to be well correlated with the mean flow evolution, as discussed in section 3 . The coherent oscillation of the zonal wind and eddy momentum fluxes suggests that a barotropic governor mechanism (James and Gray 1986; James 1987) is at work, in which the increases in horizontal shear caused by the acceleration of the jet reduce the growth rates of baroclinic instability.

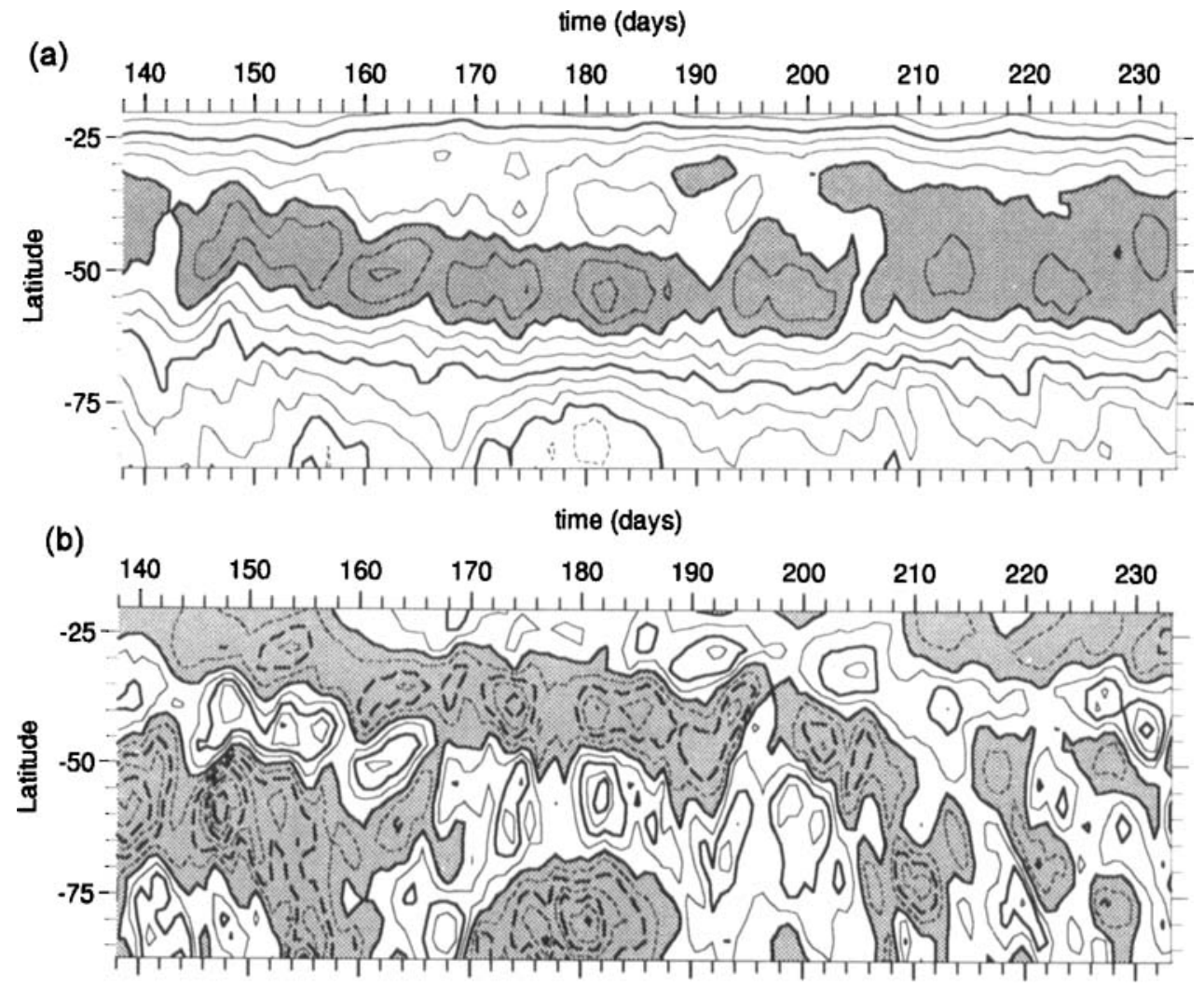

Figure 14. As Fig. 13, but showing zonal-mean zonal wind. (a) Total, contour interval $5 \mathrm{~m} \mathrm{~s}^{-1}$, shading $>30 \mathrm{~m} \mathrm{~s}^{-1}$; (b) transient, contour interval $2 \mathrm{~m} \mathrm{~s}^{-1}$, negative values shaded. In both cases negative contours are dashed. 
There are of course many limitations in using a model to study atmospheric processes. At the lower boundary this model has three levels in the first kilometre, which is not sufficient to describe accurately the antarctic boundary layer. The importance of accurately describing the boundary layer has been investigated by Pettré et al. (1990), who carried out some model experiments in which they adjusted the boundary-layer parametrization. They found that including the downslope buoyancy forces in their parametrization increased the drainage flow, and resulted in a $2 \mathrm{~K}$ warming over Antarctica. Although the details of the boundary-layer mechanics clearly modify the strength of the drainage flow it is hoped that the mechanisms of the upper-tropospheric response are insensitive to these details.

The horizontal resolution is also a limitation. That used here (T42) is sufficient to represent the broad structure of the energy containing eddies in the storm tracks, but cannot resolve details such as frontal collapse. Smaller-scale systems such as polar lows cannot be resolved. The representation of moist processes is also open to question. All these factors probably contribute to the errors in the representation of the storm track noted in section 6 . In spite of these limitations the model reproduces the essential features of the antarctic climate, namely a strong drainage flow and a vigorous storm track off the coast.

The meridional circulation associated with the drainage flow generates strong forcing of the zonal-mean momentum in the upper troposphere, up to $15 \mathrm{~m} \mathrm{~s}^{-1}$ day $^{-1}$, above the edge of the continent. The isentropic EP-flux diagnostics show that this forcing is balanced by momentum fluxes associated with synoptic-scale disturbances. The two dominant eddy-forcing terms, the eddy vertical momentum stresses and the meridional momentum flux, are of the same order of magnitude in this model, with the former being the larger of the two. The vertical EP-flux convergence is associated with a geostrophic mass flux (Johnson 1989; also discussed in section 3) and has, in the absence of significant orographic form drag, a near-zero vertical integral. This implies that asymmetries in the flow are important not only in the upper troposphere, as predicted by Egger (1985) and James (1989), but also in the boundary layer. The momentum balance in the boundary layer, in the zonal mean on isentropic surfaces, is between the drainage flow and vertical eddy transports, rather than being dominated by a balance between friction and the drainage flow. Here friction only accounts for about $1 / 3$ of the momentum source needed to balance the Coriolis torque generated by the drainage flow.

The dynamics responsible for the horizontal EP fluxes have been clarified by $\mathbf{v}$. Detten and Egger (1994). They show that the antarctic orography has an important role in enabling synoptic eddies from the storm tracks to propagate to high latitudes. The relative importance of the two mechanisms, horizontal versus vertical fluxes, cannot be accurately assessed from a single season of a single GCM, but our results suggest that both are important.

In general, the convergence of the vertical component of the EP flux is larger than that of the horizontal component. Nevertheless, the manipulations in section 3 and the above results make it clear that the horizontal component plays a crucial role in the momentum equation. The transformed equations clarify the link between surface friction and the horizontal eddy fluxes. $f \hat{v}_{\mathrm{ag}}$ acts to transfer momentum vertically, giving little net meridional transport since $\int \hat{v}_{\mathrm{ag}} \mathrm{d} p \approx 0$. At the surface the dominant terms in the transformed momentum equation will be friction and $f \hat{v}_{\mathrm{ag}}$. In the upper troposphere $f \hat{b}_{\mathrm{ag}}$ must be balanced by $D_{\phi}$. Thus, surface friction must be balanced by convergence and divergence of the horizontal eddy momentum flux $\widehat{u^{*} v^{*}}$ in the upper troposphere. The same argument applies in the Eulerian isobaric zonal-mean picture. 


\section{ACKNOWLEDGEMENTS}

This work was funded by the British Antarctic Survey, as part of the Second Antarctic Special Topic Program, and the UK Universities Global Atmospheric Modelling Programme. Thanks to J. Egger and an anonymous referee for constructive criticism of an earlier manuscript.

Alexander, R. C. and Mobley, R. L. 1976

Andrews, D. G.

Andrews, D. G. and McIntyre, M. E. 1976

Andrews, D. G., Holton, J. R. and Leovy, C. B.

Ball, F. K.

1987

1960

Barnes, J. R. and Young, R. E.

1992

Blackmon, M. L., Wallace, J. M., Lau, N-C. and Mullen, S. L.

Bromwich, D. H., Parish, J. R. and Zorman, C. A.

Bryan, $\mathbf{K}$.

1987

v. Detton, Y. and Egger, J.

1994

Dunkerton, T. J., Hsu, C.-P. F. and McIntyre, M. E.

Edmon, H. J., Hoskins, B. J. and 1980

Egger, J. McIntyre, M. E.

Eliassen, A.

Hoskins, B. J.

Hoskins, B. J. and Valdes, P. J.

Hoskins, B. J., James, I. N. and White, G. H.

Hoskins, B. J., McIntyre, M. E. and 1985 Robertson, A. W.

Iwasaki, $\mathbf{T}$.

1989

1990

1992

James, I. N.

1987

1989

\section{REFERENCES}

Monthly average sea-surface temperatures and ice-pack limits on a $1^{\circ}$ global grid. Mon. Weather Rev., 104, 143-148

A finite-amplitude Eliassen-Palm theorem in isentropic coordinates. J. Atmos. Sci., 40, 1877-1883

Planetary waves in horizontal and vertical shear: The generalized Eliassen-Palm relation and the mean zonal acceleration. J. Atmos. Sci., 33, 2031-2048

Middle atmosphere dynamics. International geophysics series, 40. Academic Press Inc.

'Winds on the ice slopes of Antarctica'. Pp. 9-16 in Antarctic meteorology: Proceedings of the symposium, Melbourne 1959. Pergamon, New York

Nonlinear baroclinic instability on the sphere: multiple life cycles with surface drag and thermal damping. J. Atmos. Sci., 49, 861-878

An observational study of the Northern Hemisphere wintertime circulation. J. Atmos. Sci., 34, 1040-1053

The confluence zone of the intense katabatic winds at Terra Nova Bay, Antarctica, as derived from airborne sastrugi surveys and mesoscaie numerical modeling. J. Geophys. Res. A, 95, (D5), 5495-5509

Potential vorticity in models of the ocean circulation. $Q . J . R$. Meteorol. Soc., 113, 713-734

Topographic wave modification and the angular momentum balance of the Antarctic troposphere. Part II: Baroclinic flows. J. Atmos. Sci., (in press)

Some Eulerian and Lagrangian diagnostics for a model stratospheric warming. J. Atmos. Sci., 38, 819-843

Eliassen-Palm cross-sections for the troposphere. J. Atmos. Sci., 37, 2600-2616

Slope winds and the axisymmetric circulation over Antarctica. J. Atmos. Sci., 42, 1859-1867

Slow thermally or frictionally controlled meridional circulation in a circular vortex. Astrophys. Norv., 5, 19-60

Towards a PV- $\theta$ view of the general circulation. Tellus, 43, 27-35

On the existence of storm-tracks J. Atmos. Sci. , 47, 1854-1864

The shape, propagation and mean-flow interaction of large scale weather systems. J. Atmos. Sci., 40, 1595-1612

On the use and significance of isentropic potential-vorticity maps. Q. J. R. Meteorol. Soc., 111, 877-946. Also 113, $402-404$

A diagnostic formulation for wave-mean flow interactions and Lagrangian-mean circulation with a hybrid vertical coordinate of pressure and isentropes. J. Meteorol. Soc. Japan, 67, 293-311

Lagrangian-mean circulation and wave-mean flow interactions of Eady's baroclinic instability waves. J. Meteorol. Soc. Japan, 68, 347-355

General circulation diagnosis in the pressure-isentrope hybrid vertical coordinate. J. Meteorol. Soc. Japan, 70, 673-687

Suppression of baroclinic instability in horizontally sheared flows. J. Atmos. Sci. , 44, 3710-3720

Antarctic drainage flow: implications for hemispheric fow on the southern hemisphere. Antarctic Science, 1, 279-290 
James, I. N.

1993

James, I. N. and Anderson, D. L. T. 1984

James, I. N. and Gray, L. J.

1986

Johnson, D. R.

Lorenz, E. N.

MacVean, M. K.

Morcrette, J-J.

1990

Palmer, T. N., Shutts, G. J. and Swinbank, R.

Palmer, T. N., Brankovic, Č., Molteni, F. and Tibaldi, S.

Parish, T. R.

1982

Parish, T. R. and Bromwich, D. H. 1986

Pettré, P., Renaud, M. F., Renaud, R., Déqué, M., Planton, S. and André, J. C Pfeffer, R. L.

Plumb, R. A.

Randel, W. J.

Randel, W. J. and Stanford, J. L.

1985

Schwerdtfeger, W

1984

Shaw, Sir Napier

1930

Simmons, A. J. and Burridge, D. M. 1981

Slingo, J. M.

1987

Slingo, J.M., Blackburn, M. and $\mathrm{He}, \mathrm{Y}$.

Thuburn, J.

1991

Tiedtke, M., Heckley, W. A. and Slingo, J. M.
The antarctic circulation in the Universities Global Atmospheric Modelling Project (UGAMP) model. In University research in Antarctica 1989-92. Ed. R. B. Heywood. British Antarctic Survey, Cambridge, England

The seasonal mean flow and distribution of large-scale weather systems in the southern hemisphere: the effects of moisture transports. Q. J. R. Meteorol. Soc., 110, 943-966

Concerning the effect of surface drag on the circulation of a baroclinic planetary atmosphere. Q.J.R. Meteorol. Soc., $112,1231-1250$

The forcing and maintenance of the global monsoonal circulation: an isentropic analysis. Advances in Geophys., 31. Academic Press, New York

Available potential energy and the maintenance of the general circulation. Tellus, 7, 157-167

The effects of horizontal diffusion on baroclinic development in a spectral model. Q.J. R. Meteorol. Soc., 109, 771783

Impact of changes to the radiation transfer parameterisations plus cloud optical properties in the ECMWF model. Mon. Weather Rev., 118, 847-873.

Alleviation of asystematic westerly bias in general circulation and numerical weather prediction models through an orographic gravity wave drag parametrization. Q.J. R. Meteorol. Soc., 112, 1001-1039

Extended-range predictions with ECMWF models: Interannual variability in operational model intergations. $Q$.J. R. Meteorol. Soc., 116, 799-834

Surface airflow over East Antarctica. Mon. Weather Rev., 110, $84-90$

The inversion wind pattern over West Antarctica. Mon. Weather Rev., 114, 849-860

The surface wind field over the Antarctic ice sheets. Nature, $328,51-54$

Study of the influence of katabatic flows on the antarctic circulation using GCM simulations. Meteorol Atmos. Phys., 43, 187-195

A study of eddy-induced fluctuations of the zonal-mean wind using conventional and transformed Eulerian diagnostics. J. Atmos. Sci., 49, 1036-1050

Eddy fluxes of conserved quantities by small amplitude waves. J. Atmos. Sci., 36, 1699-1704

'Global atmospheric circulation statistics, 1000-1 mb'. NCAR Technical Note, 295+STR. (Available from: Information services, NCAR, Boulder, Colorado)

An observational study of medium-scale wave dynamics in the Southern Hemisphere summer. Part II: Stationarytransient wave interference. J. Atmos. Sci., 42, 1189-1197

Weather and climate of the antarctic. Elsevier, Amsterdam

Manual of meteorology, vol. III: The physical processes of weather. Cambridge University Press

An energy and angular-momentum conserving vertical finitedifference scheme and hybrid vertical coordinates. Mon. Weather Rev., 109, 758-766

The development and verification of a cloud prediction scheme for the ECMWF model. Q. J. R. Meteorol. Soc., 113, $899-927$

1991 'Recent improvements in the physical processes of the UGAMP general circulation model'. UGAMP Technical Report, 17. (Available from: Dept. Meteorology, University of Reading, Reading, England)

Data sampling strategies for general circulation models. Q. $J$. R. Meteorol. Soc., 117, 385-397

1988 Tropical forecasting at ECMWF: The influence of physical parametrization on the mean structure of forecasts and analyses. $Q . J$. $R$. Meteorol. Soc., 114, 639-664 
Townsend, R. D. and Johnson, D. R. 1985

Trenberth, K. E.

Tung, K.-K.

Yasunari, T. and Kodama, S.

1993
A diagnostic study of the isentropic zonally averaged mass circulation during the First GARP Global Experiment. J. Atmos. Sci. , 42, 1565-1579

1991 Storm tracks in the southern hemisphere. J. Atmos. Sci., 48, 2159-2178

1986 Nongeostrophic theory of the zonally-averaged circulation. Part 1: Formulation. J. Atmos. Sci. 43, 2600-2618 arctica and planetary flow regimes in the southern hemisphere. J. Geophys. Res., 98, 13063-13070 